\title{
Post-lockdown Dynamics of COVID-19 in New York, Florida, Arizona, and Wisconsin
}

\author{
Sherry Scott ${ }^{1}$, Keisha J. Cook $^{2}$, Kamal Barley ${ }^{3}$ \\ Department of Mathematics, formerly MSOE, Department of Mathematics, Tulane University. Applied Mathematics $\mathcal{E}$
} Statistics, Stony Brook University.

\section{Abstract}

7 The COVID-19 pandemic is widely studied as it continues to threaten many populations of people especially in the USA, the leading country in terms of both deaths and cases. Although vaccines are being distributed, control and mitigation strategies must still be properly enforced. More and more reports show that the spread of COVID-19 involves infected individuals first passing through a pre-symptomatic infectious stage in addition to the incubation period and that many of the infectious individuals are asymptomatic. In this study, we design and use a mathematical model to primarily address the question of who are the main drivers of COVID-19 - the symptomatic infectious or the pre-symptomatic and asymptomatic infectious in the states of Florida, Arizona, New York, Wisconsin and the entire United States. We emphasize the benefit

\section{1. Introduction}

The coronavirus, known as COVID-19, emerged in Wuhan, China in December 2019, and has since then spread to all countries on earth. As of 12/10/2020, the United States has reported 15,535,565 cases and 291,403 deaths and although the leading country in both deaths and cases, the U.S. did not implement a national pandemic control initiative. Instead, many states and cities implemented their own initiatives at 


\section{2. Main Questions}

various times, and the transmission of COVID-19 has varied from state to state. States also implemented their own testing initiatives. Around 12-10-2020, the reported testing rates are as follows: Arizona testing at a rate of 347.7 tests per 100,000 people, Florida testing at a rate of 497.2 tests per 100,000 people, New York testing at a rate of 985.7 tests per 100,000 people, and Wisconsin testing at a rate of 562.4 tests per 100,000 people [2]. With regard to mask usage, the observed mask usage percentage in the US has increased since the beginning of the pandemic. From March 1, 2020 to May 1, 2020, the mask use percentage increased from $5 \%$ to $40 \%$. From May 1, 2020 to November 22, 2020, the mask use percentage increased from $71 \%$. Social distancing has also played a role in the transmission of COVID-19. From March 8, 2020 to April 4, 2020, the US experienced a 53\% decrease in mobility. From April 4, 2020 to June 24, 2020, the decrease in mobility changed to only -22\%. From June 24, 2020 to November 22, 2020, the mobility oscillated between $-18 \%$ and $-25 \%$ [3]. The social distancing statistics are due to the fact that different states implemented stay-at-home periods for various intervals of time. All of these factors play a part in the transmission of COVID-19 in the US as a whole, and effectively in each state. Although we do not consider all of these measures directly, we do discuss the effect that lockdown has on the number of cases and deaths. We do include mask usage, and we use a different mask compliance value for each period per state [14. To study social distancing, we fit the values for the effective contact rate for each time period per state and per time period.

Mathematical modelling can help us better understand how COVID-19 cases and mortality is affected based on when and how long lockdown periods are enforced. Modelling can also help us identify the main drivers of the disease and give insight on how best to use the standard control and mitigation strategies. We expand on the standard SIR model, which uses a system of ordinary differential equations to model disease spread through multiple compartments over time. In this study, we do not consider a time-varying system.

Prior studies consider susceptible individuals transitioning to exposed and then to infectious. We include a pre-symptomatic compartment for individuals who are infectious before the onset of symptoms. We also consider individuals who were tested, resulting in an asymptomatic or symptomatic classification, as well as the population of people who may have self-isolated after being exposed by an infected individual. It is known that over the course of the pandemic, hospitals have been highly stressed and over-crowded due to the influx of COVID-19 patients. In order to study mortality due to the pandemic, we also include compartments that keep track of the number of patients admitted to the hospital and the ICU.

We explore scenarios where lockdown is started earlier or extended past the reported date. We show that both scenarios decrease the number of deaths. We can use these type of simulations to predict future mortality populations and use the information to help with determining when lockdown periods should start and how long the lockdown period should be enforced.

Finally, in response to the increase in public discussions of a natural herd immunity approach, we use our model to run some basic scenarios for implementing such a strategy in the state of Wisconsin and the US as a whole. We simulate the number of deaths that would occur before herd immunity is achieved.

\footnotetext{
The overall purpose of this study is to explore the post-lockdown dynamics of COVID-19 and in the process, explore the notion that the main drivers of COVID19 are the asymptomatic and pre-symptomatic infectious individuals. Because both the asymptomatic and pre-symptomatic infectious spread COVID-19 without showing symptoms we group these two compartments together and compare that group to the symptomatic infectious. More specifically, we use a mathematical model to address the following questions for New York, Arizona, Florida, Wisconsin, and in some cases, the entire US. The section of the paper in which each question is addressed is given after the question.
}

- How do changes in lockdown dates (i.e., earlier or later lockdown dates) affect the number of deaths and cases? (Section 4.3.1) 
- How does the contribution of the pre-symptomatic and asymptomatic infectious individuals compare to that of the symptomatic infectious in terms of cases and deaths? (Section 4.3.2)

- If we isolate all symptomatic infectious individuals or all asymptomatic and pre-symptomatic infectious individuals, how will this impact deaths and cases? (Section 4.3.3)

- How might a minimum testing rate for pre-symptomatic and asymptomatic compare to that for the symptomatic infectious individuals in order to obtain a reproduction number below 1? (section 4.3.4)

- How many deaths will occur in a natural herd immunity simulation for Wisconsin and the entire US? (Section 4.4)

\subsection{COVID-19}

In order to assess and deliver guidelines for individuals to avoid coronavirus infection, the CDC has implemented models equipped with the many stages of the virus [1. A person who has been infected is considered pre-symptomatic (an individual who is infectious but did not show symptoms at the time of testing) or asymptomatic (a person who is infectious and does not show symptoms throughout the course of the infection). The CDC built pandemic planning scenarios varying the infectiousness of asymptomatic individuals compared to symptomatic, varying the percent of asymptomatic individuals, and varying the percentage of transmission of pre-symptomatic individuals. In this paper, we use similar methods to build our model and determine parameter values for the locations and time periods of interest. Figure 1 shows the stages of COVID-19, especially the incubation period, infectious period, pre-symptomatic period, and symptomatic period. It should be noted that a pre-symptomatic infectious individual can become either asymptomatic or symptomatic infectious.

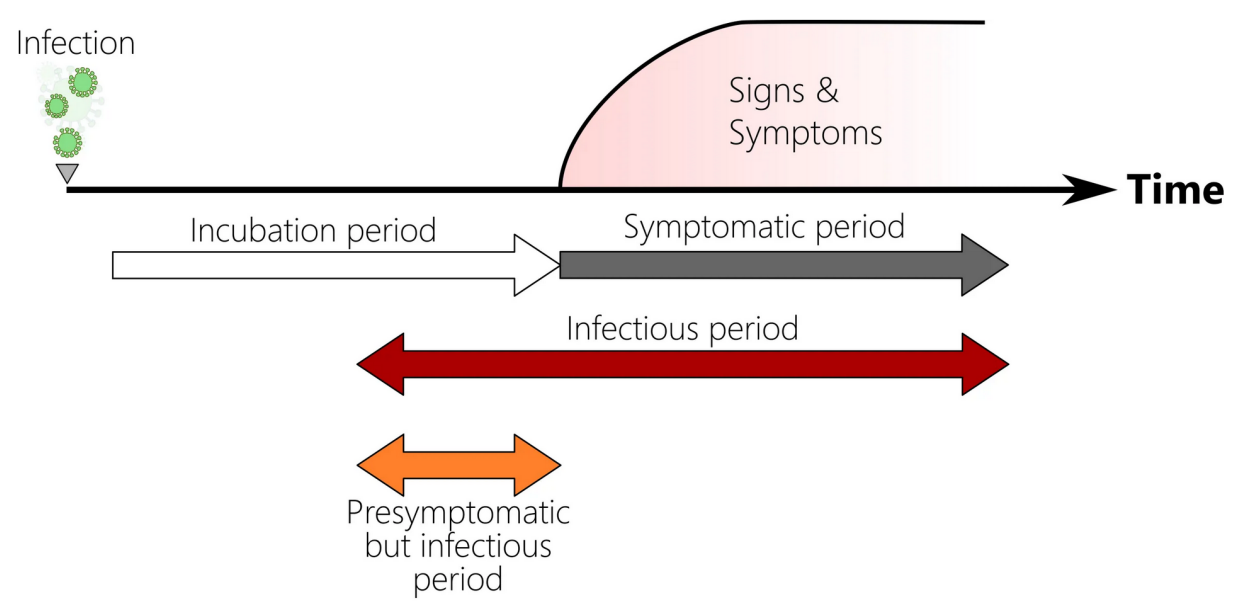

Figure 1: The Stages of COVID-19

We are interested in modeling COVID-19 in the United States as a whole, as well as focusing on four states. We consider New York (Northeast), which started as an epicenter in the US. We consider Florida (Southeast) and Arizona (Southwest) due to the fact that they became hot spots with significant numbers of daily cases and overwhelming numbers in their healthcare facilities. Lastly, we consider Wisconsin (Midwest), which became a hot spot later in the year. Table 1 shows the differences between the states with regards to the number of cumulative deaths.

Cumulative Deaths

\begin{tabular}{|c|c|c|c|c|}
\hline & Beginning of lockdown & End of lockdown & on August 11, 2020 & on November 23, 2020 \\
\hline United States & 16767 & 104803 & 164519 & 257779 \\
Arizona & 25 & 651 & 4199 & 6464 \\
Florida & 170 & 1399 & 8553 & 18085 \\
New York & 249 & 30482 & 32770 & 34339 \\
Wisconsin & 10 & 594 & 1006 & 3158 \\
\hline
\end{tabular}

Table 1: This table gives the cumulative deaths for each state and the United States. The lockdown dates depend on the state. 


\section{Methods}

3.1. Model with pre-symptomatic compartment and face masks

We use a modified Kermack-McKendrik-type epidemic (no human demography) model to better understand the dynamics of COVID-19. Since a main objective of this study is to analyze the role of infectious individuals who show no symptoms, the model (2) includes a pre-symptomatic compartment as the foundation for parameter estimation. We use a deterministic susceptible, exposed, pre-symptomatic, symptomaticallyinfectious, asymptomatically-infectious, self-isolated, hospitalized, recovered, and ICU patients modeling framework, with the classes denoted as $S(t), E(t), E_{P}(t), I(t), A(t), J(t), H(t), R(t), C(t)$ respectively; we also include $D(t)$ to track deaths. The model also includes the mitigation/control interventions of face mask usage (with a compliance parameter as well as a face mask efficacy parameter) and testing/detection implementation for each of the infectious classes.

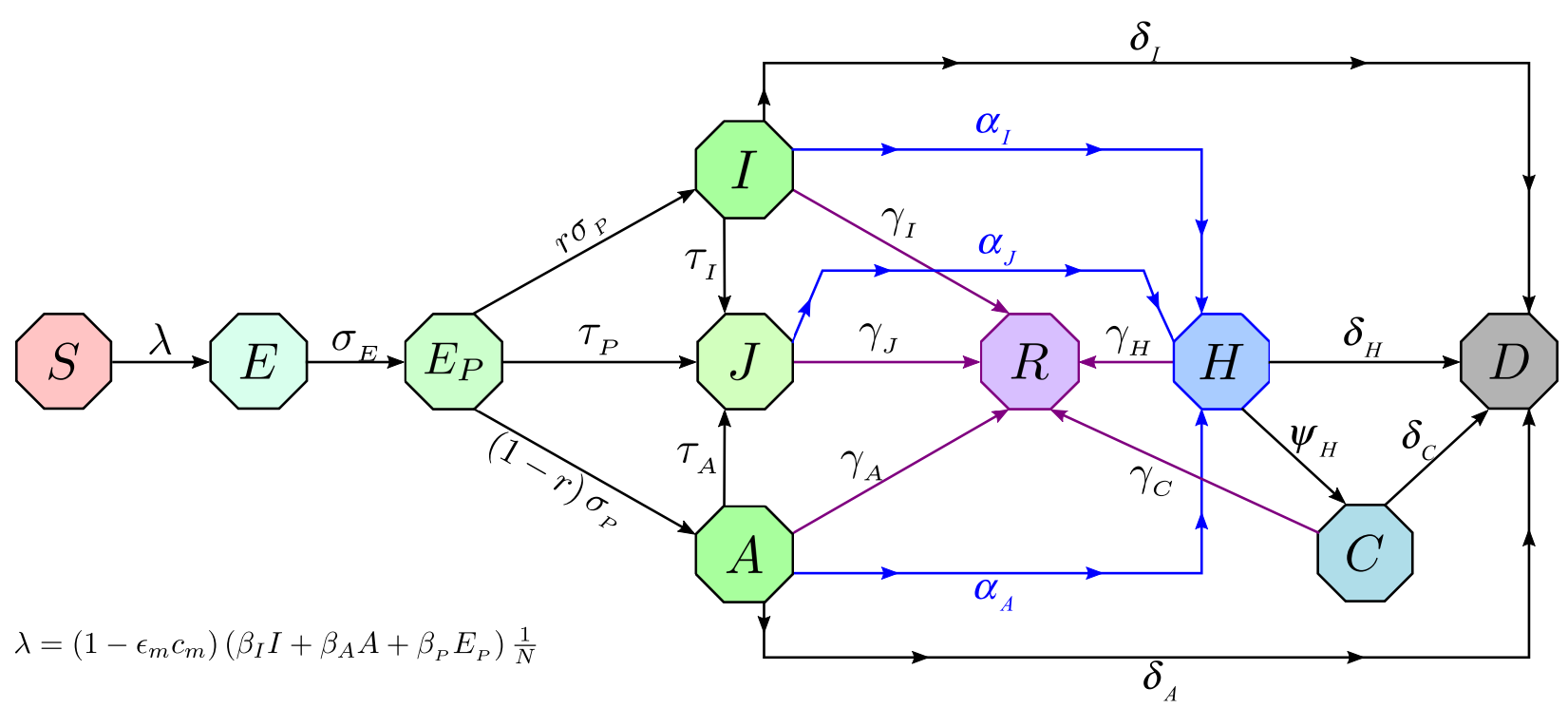

Figure 2: Schematic flow diagram of the model

\subsection{Data Collection}

We obtain the observed cumulative deaths and cases data for the states of Arizona, Florida, New York

Our model for the transmission dynamics of COVID-19 is given by the following deterministic system of non-linear differential equations. 


$$
\begin{aligned}
\frac{d S}{d t} & =-\beta_{I}\left(1-\epsilon_{m} c_{m}\right) \frac{S I}{N}-\beta_{A}\left(1-\epsilon_{m} c_{m}\right) \frac{S A}{N}-\beta_{P}\left(1-\epsilon_{m} c_{m}\right) \frac{S E_{P}}{N}=-\lambda S \\
\frac{d E}{d t} & =\beta_{I}\left(1-\epsilon_{m} c_{m}\right) \frac{S I}{N}+\beta_{A}\left(1-\epsilon_{m} c_{m}\right) \frac{S A}{N}+\beta_{P}\left(1-\epsilon_{m} c_{m}\right) \frac{S E_{P}}{N}-\sigma_{e} E=\lambda S-\sigma_{e} E \\
\frac{d E_{P}}{d t} & =\sigma_{e} E-\sigma_{p} E_{P}-\tau_{p} E_{P} \\
\frac{d I}{d t} & =r \sigma_{p} E_{P}-\alpha_{I} I-\tau_{I} I-\gamma_{I} I-\delta_{I} I \\
\frac{d A}{d t} & =(1-r) \sigma_{p} E_{P}-\alpha_{A} A-\tau_{A} A-\gamma_{A} A-\delta_{A} A \\
\frac{d H}{d t} & =\alpha_{I} I+\alpha_{A} A+\alpha_{J} J-\psi_{H} H-\gamma_{H} H-\delta_{H} H \\
\frac{d C}{d t} & =\psi_{H} H-\gamma_{C} C-\delta_{C} C \\
\frac{d J}{d t} & =\tau_{p} E_{P}+\tau_{A} A+\tau_{I} I-\alpha_{J} J-\gamma_{J} J \\
\frac{d R}{d t} & =\gamma_{I} I+\gamma_{A} A+\gamma_{C} C+\gamma_{J} J+\gamma_{H} H \\
\frac{d D}{d t} & =\delta_{A} A+\delta_{I} I+\delta_{H} H+\delta_{C} C
\end{aligned}
$$

where $N(t)=S+E+E P+I+A+H+J+C+R$ is the total population at time $t$. Let $\epsilon_{m}$ be the efficacy of face masks to prevent transmission and acquisition of infection, $c_{m}$ the compliance in face mask usage in the community $\left(0<c_{m} \leq 1\right), \sigma$ the progression rate from exposed $(E)$ to infectious classes $(I$ or $A), r$ the fraction of exposed individuals who show clinical symptoms at the end of the incubation period, $\tau_{I}$ the rate self-quarantined symptomatically-infectious humans self-isolate, $\gamma_{I}, \gamma_{A}, \gamma_{J}, \gamma_{H}, \gamma_{C}$ the recovery rates for the subscripted population, $\psi_{H}$ the rate of ICU admission for hospitalized individuals, $\alpha_{I}$ the hospitalization rate for symptomatically-infectious individuals, $\alpha_{A}$ the hospitalization rate for asymptomatically-infectious individuals, $\delta_{I}$ the disease-induced mortality rate for symptomatically-infectious individuals, $\delta_{A}$ the diseaseinduced mortality rate for asymptomatically-infectious individuals, $\delta_{H}$ the disease-induced mortality rate for hospitalized individuals, $\delta_{C}$ the disease-induced mortality rate individuals in ICU. We assume that hospitalized individuals do not come in contact with the general population.

157 4. Results

\subsection{Computation of Reproduction Numbers}

The basic reproduction number $\mathcal{R}_{0}$ is the average number of secondary infections produced when one infected individual is introduced into a host population of susceptible individuals. For our model's $\mathcal{R}_{0}$, we have $\mathcal{R}_{0}=\mathcal{R}_{0 A}+\mathcal{R}_{0 I}+\mathcal{R}_{0 P}$ where

$$
\begin{gathered}
\mathcal{R}_{0 A}=\frac{\beta_{A}(1-r)}{\alpha_{A}+\gamma_{A}+\delta_{A}} \\
\mathcal{R}_{0 I}=\frac{\beta_{I} r}{\alpha_{I}+\gamma_{I}+\delta_{I}}
\end{gathered}
$$

and

$$
\mathcal{R}_{0 P}=\frac{\beta_{P}}{\sigma_{P}} .
$$

159 The control reproduction number $\mathcal{R}_{C}$ is the average number of new cases generated by a typical in160 fectious individual introduced into a host population of susceptible individuals with some control measures/interventions in place. Using the next generation operator method and notation found in [17] we have the following computation for the control reproduction number $\mathcal{R}_{C}$. If we take the column vector

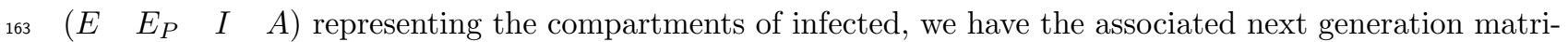
164 ces, $F$ and $V$, for the new infection terms and the transition terms are given respectively as 


$$
\mathcal{F}=\left[\begin{array}{cccc}
0 & \left(1-\epsilon_{m} c_{m}\right) \beta_{P} & \left(1-\epsilon_{m} c_{m}\right) \beta_{I} & \left(1-\epsilon_{m} c_{m}\right) \beta_{A} \\
0 & 0 & 0 & 0 \\
0 & 0 & 0 & 0 \\
0 & 0 & 0 & 0
\end{array}\right] \text { and } \mathcal{V}=\left[\begin{array}{cccc}
\sigma_{e} & 0 & 0 & 0 \\
-\sigma_{e} & G_{1} & 0 & 0 \\
0 & -r \sigma_{P} & G_{2} & 0 \\
0 & -(1-r) \sigma_{P} & 0 & G_{3}
\end{array}\right]
$$

where, $G_{1}=\sigma_{P}+\tau_{P}, G_{2}=\alpha_{I}+\gamma_{I}+\delta_{I}+\tau_{I}, G_{3}=\alpha_{A}+\gamma_{A}+\delta_{A}+\tau_{A} . \mathcal{R}_{C}$ is the spectral radius of the next generation matrix given by,

$$
\mathcal{R}_{C}=\rho\left(F V^{-1}\right)=\mathcal{R}_{I}+\mathcal{R}_{A}+\mathcal{R}_{P}
$$

where $\mathcal{R}_{I}=\frac{\left(1-\epsilon_{m} c_{m}\right) \beta_{I} r \sigma_{P}}{G_{1} G_{2}}, \mathcal{R}_{A}=\frac{\left(1-\epsilon_{m} c_{m}\right)(1-r) \beta_{A} \sigma_{P}}{G_{1} G_{3}}$, and $\mathcal{R}_{P}=\frac{\left(1-\epsilon_{m} c_{m}\right) \beta_{P}}{G_{1}}$. In absence of interventions, i.e. with $\tau_{I}=\tau_{A}=\tau_{P}=\tau_{A}=\epsilon_{m}=c_{m}=0$, we get the basic reproduction number for model (1), $\mathcal{R}_{0}$, as given earlier.

Theorem 1. The disease-free equilibrium (DFE) of the model 1 is locally-asymptotically stable if $\mathcal{R}_{C}<1$, and unstable if $\mathcal{R}_{C}>1$

A basic implication of Theorem 1 is that in order to control the COVID-19 outbreak (to not generate more), it suffices to keep $\mathcal{R}_{C}<1$.

\subsection{Goodness of Fit}

We analyze how well our model fits the data from John Hopkins using the T-Test in $R$. The T-Test measures the difference between two sets of continuous data. We want to compare two sample means to determine if there is a statistically significant difference between the model and the data. We use a paired T-test where each vector has the same amount of entries and the values are taken from the same independent variable. The T-Test assumes that the data in each vector is normally distributed and that they have approximately equal variances. To test for normality, we use the Shapiro-Wilk Test in $R$. The null hypothesis for this test is that the values in the vector are normally distributed. If the p-value is greater than 0.05 , then the distribution of the values in the vector are not significantly different from the normal distribution. I.e. we fail to reject the null hypothesis. We can visualize this using a Q-Q plot. We assume normality if all the points fall along the reference line. We then check the variances of the values in the two vectors using the standard variance equation from probability. Let the null hypothesis for the T-Test be that the difference between the two groups is 0 . The T-statistic is defined as

$$
T=\frac{\overline{x_{1}}-\overline{x_{2}}}{\sqrt{\left(s_{1}^{2} / n_{1}\right)+\left(s_{2}^{2} / n_{2}\right)}}
$$

where, $\overline{x_{1}}$ is the mean of the data, $\overline{x_{s}}$ is the mean of the model values, $s_{1}^{2}$ is the variance of the data, $s_{2}^{2}$ is the variance of the model values, $n_{1}$ is the sample size of the data, and $n_{2}$ is the sample size of the model values. We conclude that the model for each state, cumulative death population, during the lockdown period does indeed fit the data. Table 2 shows the resulting p-values of the T-Test for each state and the United States as a whole during the lockdown period. The goodness of fit plots can be found in the appendix (section 6.3) in Figures 17, 18, 19, 20, and 21.

\begin{tabular}{|c|c|c|c|c|}
\hline & Daily p-value & Daily T-statistic & Cumulative p-value & Cumulative T-statistic \\
\hline United States & 0.9722 & 0.035037 & 0.7839 & 0.27581 \\
Florida & 0.9083 & 0.11621 & 0.4947 & 0.69165 \\
Arizona & 0.6002 & -0.52796 & 0.0004622 & 3.7845 \\
New York & 0.8781 & -0.15396 & 0.007702 & 2.749 \\
Wisconsin & 0.02353 & -2.323 & $2.2 \mathrm{e}-16$ & -17.953 \\
\hline
\end{tabular}

Table 2: Goodness of Fit for the T-Test. This table gives the p-values and T-statistic for each test for each state and the United States during the lockdown period. The daily column denotes the model vs the data of the daily deaths. The cumulative column denotes the model vs the data of the cumulative deaths. 


\subsection{Simulated Epidemics}

We fit our model using the cumulative and daily mortality data for Florida, Arizona, Wisconsin, and New York over the pre-lockdown, lockdown, and post-lockdown periods based on the state's respective decisions.
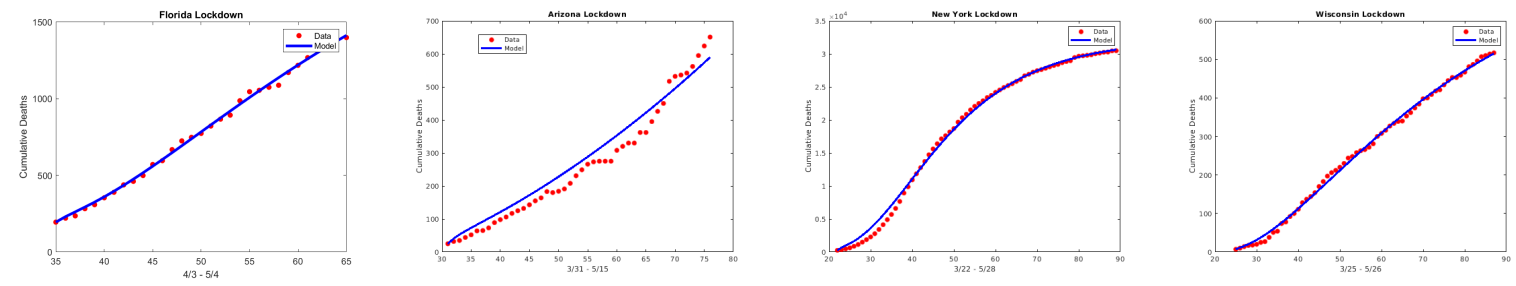

Figure 3: Data fitting of the model using COVID-19 mortality data for the states of Florida, Arizona, New York and Wisconsin during their respective lockdown periods. The plots show the model (blue) against the data (red).

The state variables and the parameters for our model are given in the Appendix in Tables 3 and 4
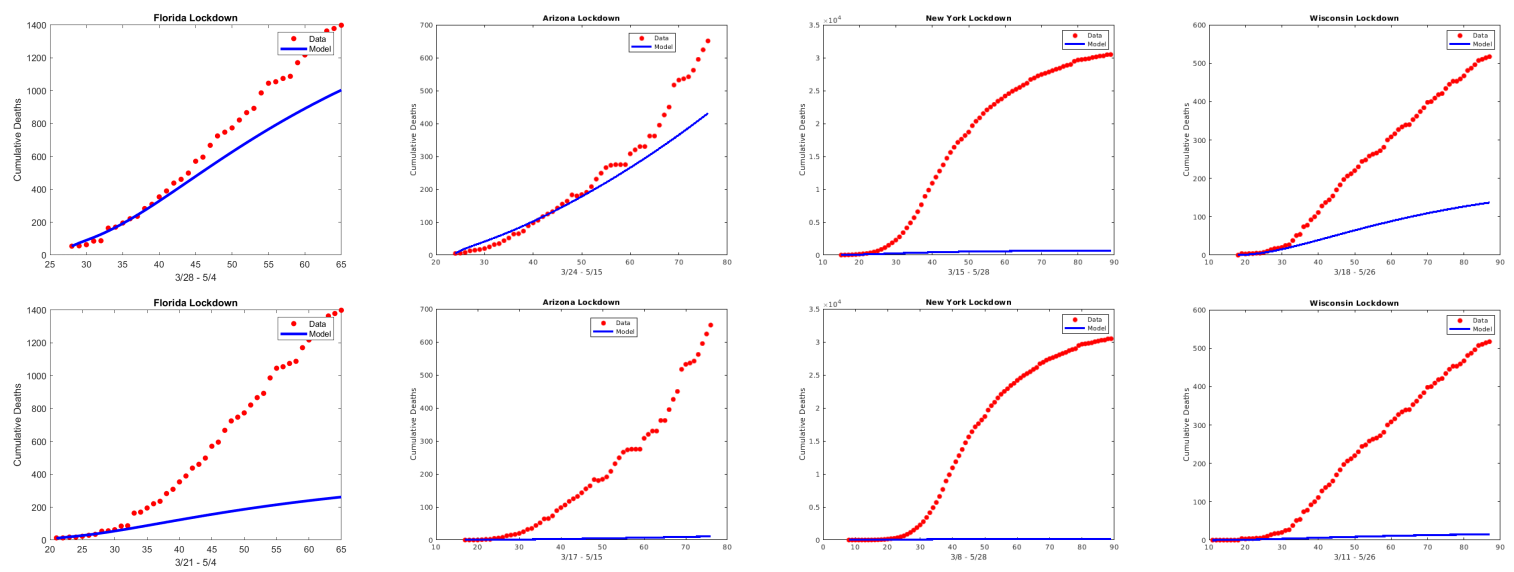

Figure 4: Row 1: The cumulative deaths for each state if lockdown began 1 week earlier. Row 2: The cumulative deaths for each state if lockdown began 2 weeks earlier.

In general, an earlier lockdown also results in a decrease in daily cases for both the symptomatic and asymptomatic/pre-symptomatic compartments, as shown in the daily cases plots for Arizona and the entire US in Figure 5. 

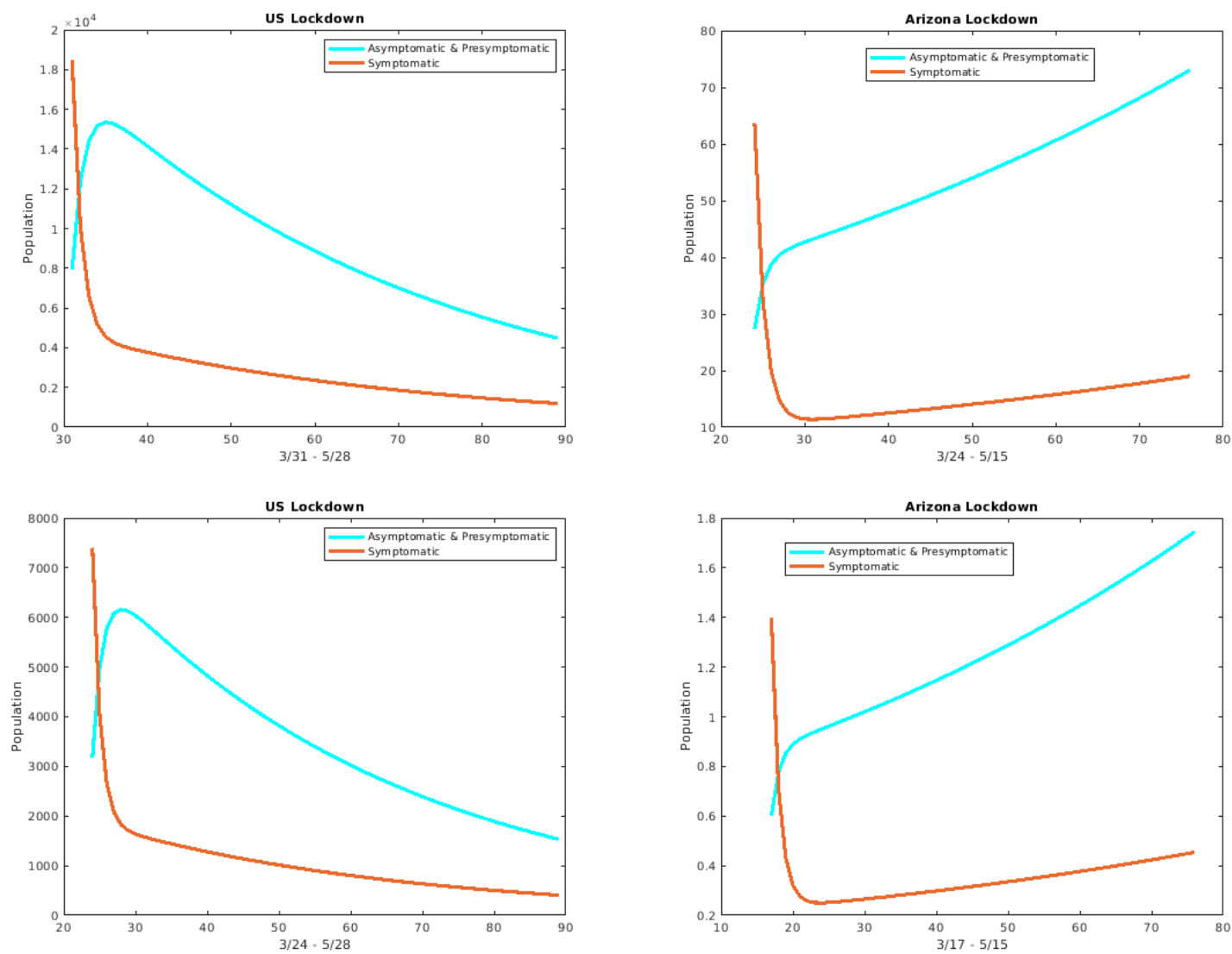

Figure 5: Daily cases for the asymptomatic/pre-symptomatic individuals (cyan curve) versus the symptomatic individuals (red curve) for the entire US and Arizona. Row 1: If lockdown began 1 week earlier. Row 2: If lockdown began 2 weeks earlier.

On the other hand, if we use the original lockdown start dates but extend the lockdown periods by 2 and 4 weeks for each state, we again see a decrease in cumulative deaths as shown in Figure 6 .
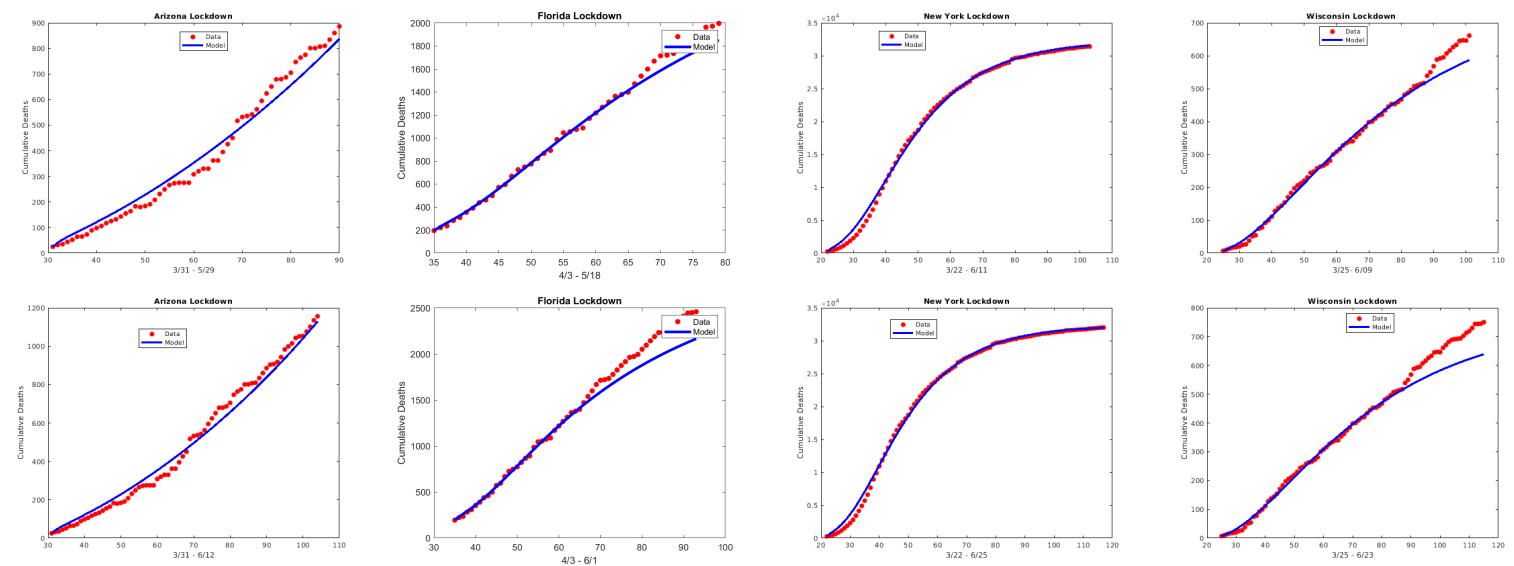

Figure 6: Row 1: The cumulative deaths for each state if lockdown was lifted 2 weeks later. Row 2: The cumulative deaths for each state if lockdown was lifted 4 weeks later.

These varying lockdown dates scenarios can be used to assess the efficacy of lockdown periods and to guide the manner in which the lockdown periods are implemented. For example, one interesting lockdown scenario is to consider what would happen if the entire U.S. implemented a lockdown during the month of December 2020 for two weeks. In order to simulate such a strategy we reduce the contact rate parameters - i.e., the betas - by $40 \%$ [1] and run the simulation forward. As shown in Figure 7 below, the simulations give that such a lockdown would result in a marked decrease in the number of deaths. 

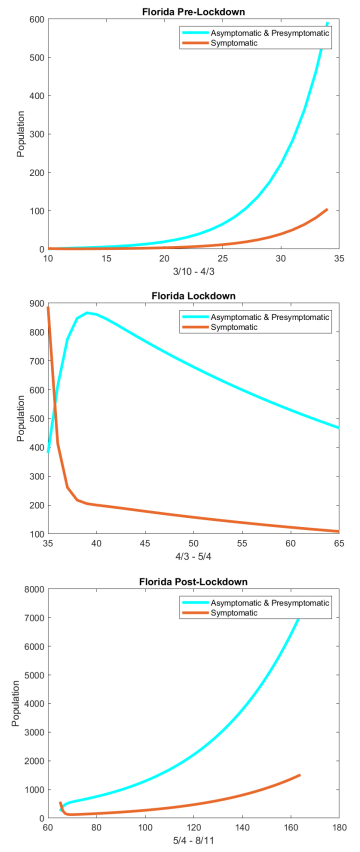
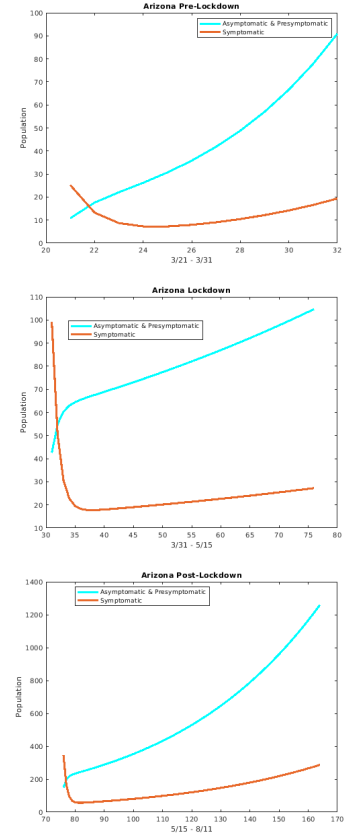

US Postlockdown

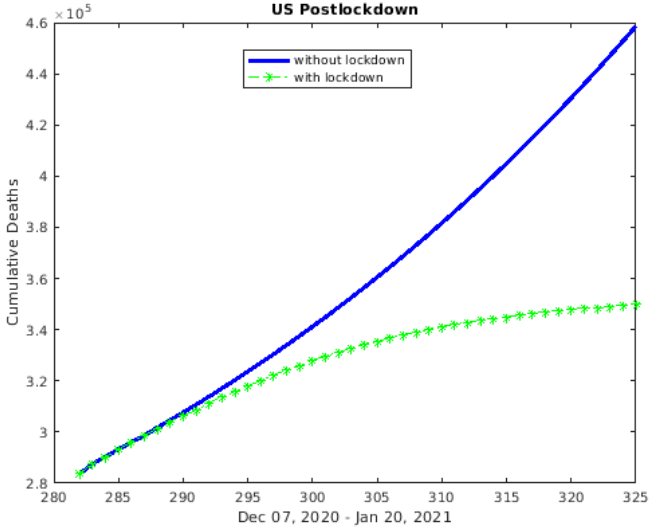

Figure 7: Cumulative deaths in US with lockdown in December (green) versus without lockdown (blue)

Figure 8: The plots show the pre-symptomatic and asymptomatic versus symptomatic individuals for each state during their respective time period. Row 1: Pre-lockdown period. Row 2: Lockdown period. Row 3: Post-lockdown period.
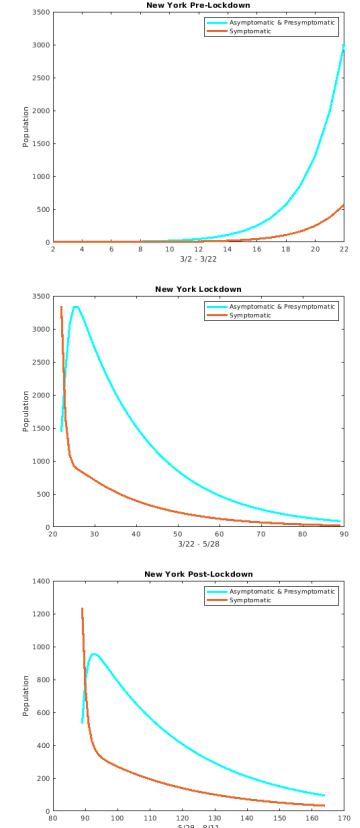
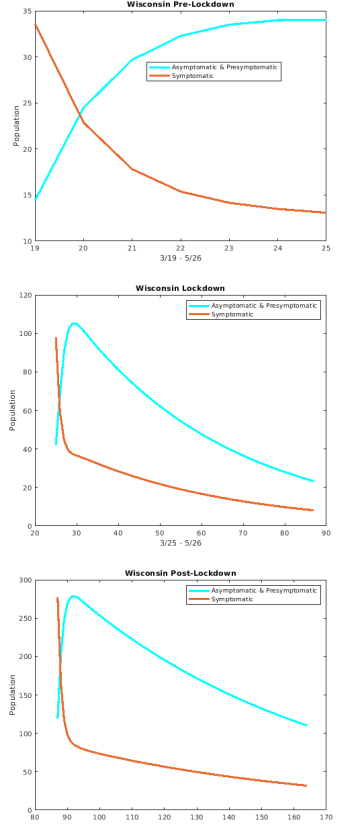

The parameter $\beta_{I}$ represents the contacts by the symptomatically infectious individuals that could transmit the disease. By setting $\beta_{I}=0$, we eliminate that transmission and focus on the effect of the pre-symptomatic and asymptomatic individuals. Similarly, fixing $\beta_{A}=\beta_{P}=0$, targets the effect of the symptomatically infectious. In order to further discern the role of the asymptomatic and pre-symptomatic infectious versus that of the symptomatic infectious, we consider these zero contact rate scenarios in terms of the number of daily cases for all of the states and the entire US. In Figure 9, we show the results for the pre-lockdown and post-lockdown periods and for those periods, we again get that the larger contribution comes from the asymptomatic and pre-symptomatic individuals. For the lockdown period we obtain mixed results and these are considered in the appendix (Figure 16). 

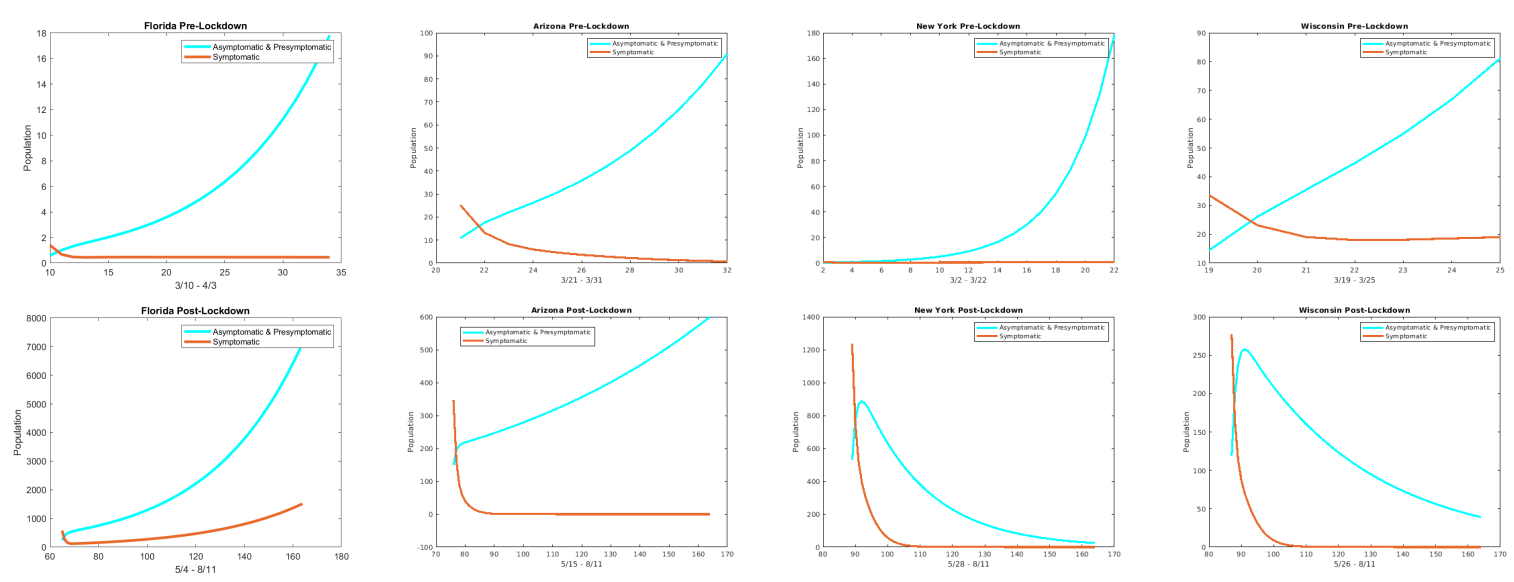

Figure 9: The plots show the daily cases for each state with $\beta_{I}=0$ (cyan curve) and with $\beta_{A}=\beta_{P}=0$ (red curve) during their respective Row 1: Pre-lockdown period and Row 2: Post-lockdown period.
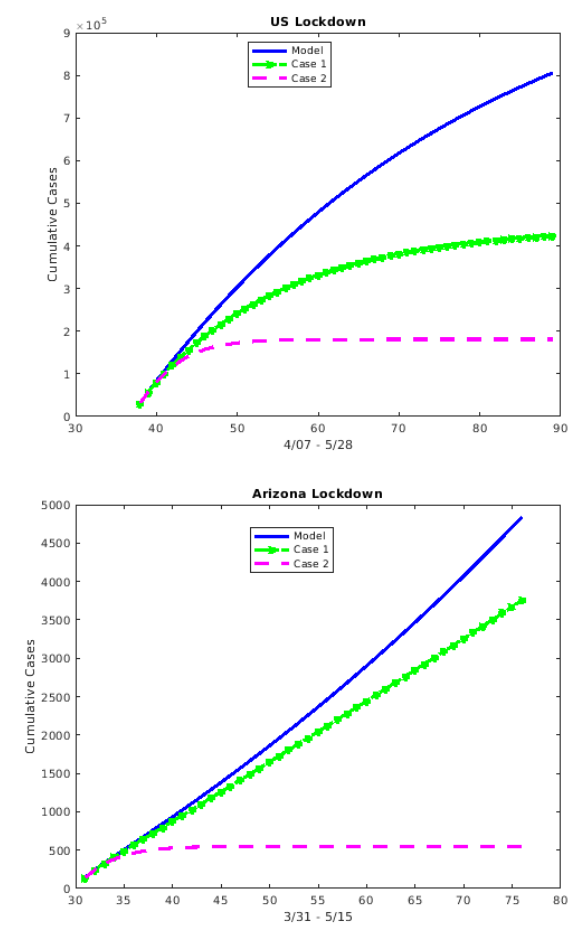
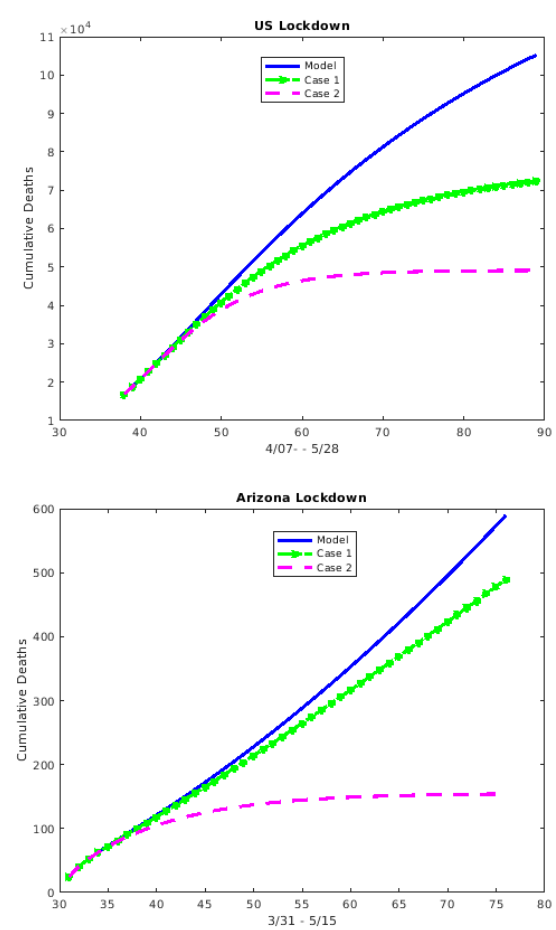

Figure 10: The cumulative cases and deaths for the US and Arizona over the respective lockdown period where Case $\mathbf{1}$ corresponds to $\beta_{I}=0$ (green curve; so focuses on the impact of asymptomatic/pre-symptomatic individuals) and Case 2 corresponds to $\beta_{A}=\beta_{P}=0$ (magenta curve; so focuses on the impact of symptomatic individuals)

\subsubsection{Isolating Symptomatic and Asymptomatic/Pre-symptomatic}

Recall that the $\tau$ values give the rate for testing/detection of infected individuals and subsequent selfisolation. In this section we compare and contrast the effect of isolating the symptomatic against the effect of isolating the asymptomatic and pre-symptomatic by changing the appropriate $\tau$ values. For example, to address the effect of isolating symptomatic infectious individuals, we can increase the value of $\tau_{I}$ (the rate that symptomatically infectious individuals self-isolate) while keeping all other rates the same. We consider this scenario for the entire US and find that when $\tau_{I}$ increases (so that the number of symptomatically infectious individuals that are self-isolated and taken out of the general population increases), the number of cumulative deaths decreases by as much as $12.5 \%$. Alternatively, if the rate of self-isolation for the asymptomatic and pre-symptomatic infectious individuals $-\tau_{A}$ and $\tau_{P}$ respectively - are increased and the $\tau_{I}$ is kept at the baseline value for the US, the number of cumulative deaths decreases by about $35 \%$ at the end of the period - see Figure 11. Thus, the decrease is more pronounced when the $\tau_{A}$ and $\tau_{P}$ are increased than when the $\tau_{I}$ is increased which indicates that the asymptomatic and pre-symptomatic have more impact. Figure 12 focuses on the difference in impact with $50 \%$ increase in testing and shows that the asmptomatic 
We can also compare the effect of these type of testing scenarios for different states. For example, although Wisconsin and Florida had similar testing rates around 12/10/2020, we find that a 50\% increase
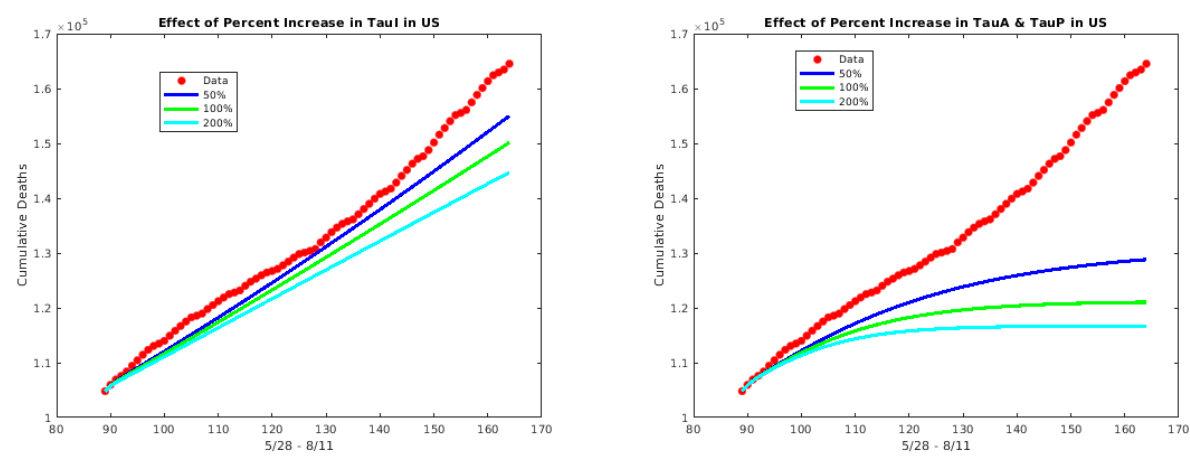

Figure 11: The effect of an increase in testing of symptomatic individuals versus asymptomatic and pre-symptomatic individuals on the cumulative deaths in the US during post-lockdown period 5/15 to $8 / 11$.

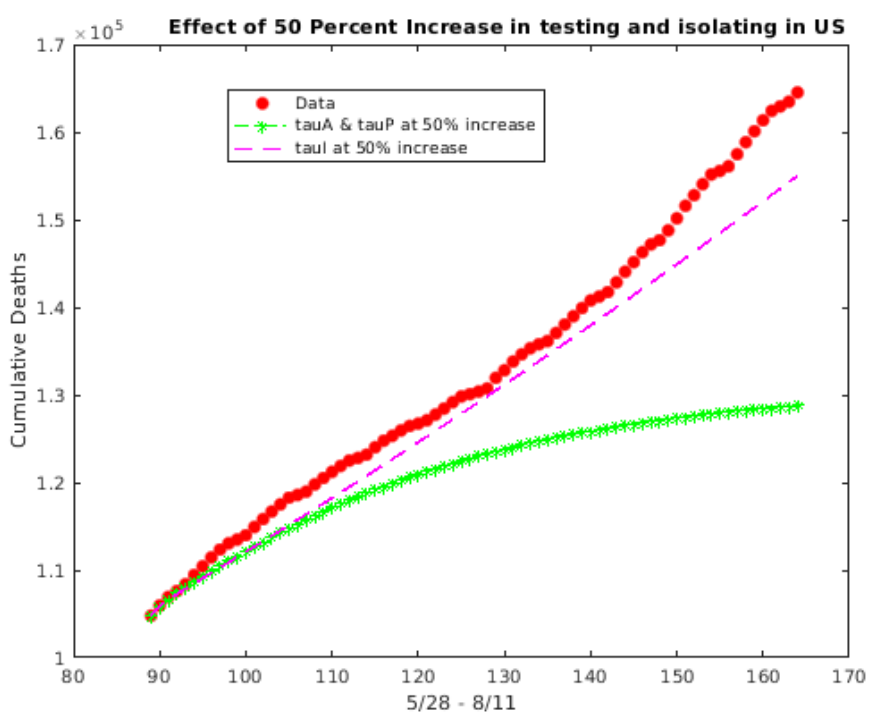

Figure 12: The effect of a $50 \%$ increase in testing of asymptomatic and pre-symptomatic versus symptomatic on the cumulative deaths in the US during post-lockdown period $5 / 28$ to $8 / 11$.
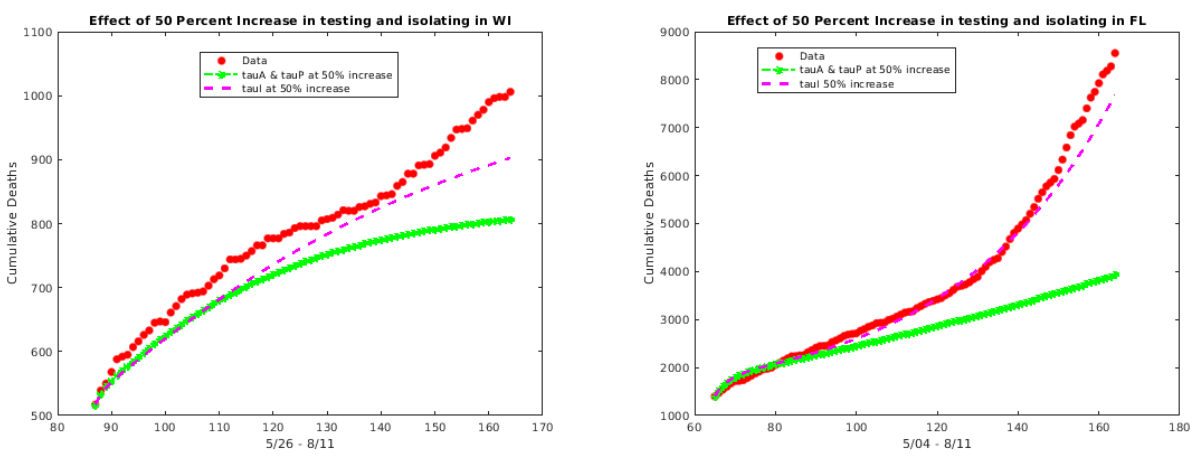

Figure 13: The effect of a 50 percent increase in testing of asymptomatic and pre-symptomatic (green curve) versus symptomatic (magenta curve) individuals on the cumulative deaths for Wisconsin and Florida.

\section{4.3.4. Minimum testing needed}

In this section we consider the question concerning the minimum rate of testing/detection required to

${ }_{253}$ lower the control reproduction number, $\mathcal{R}_{C}$ below one. This question is more difficult to answer directly,

${ }_{254}$ but we can begin to get at an answer by again considering the effect of increasing testing. For example, for

255 the entire US from May 28 to Aug 11 (after lifting of lockdown) if the testing rate for asymptomatic and 
pre-symptomatic is 0.2 - that is, if $\tau_{A}$ and $\tau_{P}$ are both set to 0.2 , while $\tau_{I}=0.4$, then the reproduction control number $\mathcal{R}_{C}$ is 1.0346 (so just above 1 ). If $\tau_{A}$ and $\tau_{P}$ are bumped above 0.2 , for example to 0.25 , while $\tau_{I}$ remains at 0.4 , then $\mathcal{R}_{C}$ falls below 1 - to 0.95178 - and the resulting decrease in cumulative deaths and daily cases is shown in Figure 14. To get a better appreciation of the impact of testing for asymptomatic and pre-symptomatic cases, note that if $\tau_{A}$ and $\tau_{P}$ are kept at their baseline fitted values, then $\tau_{I}$ must be increased to 1.7 to get $\mathcal{R}_{C}=0.9945$. Of course, the challenge with all of these testing scenarios is that the testing of asymptomatic and pre-symptomatic individuals is more difficult since those individuals do not have symptoms, so in order to achieve these testing rates, widespread and frequent testing should be implemented.
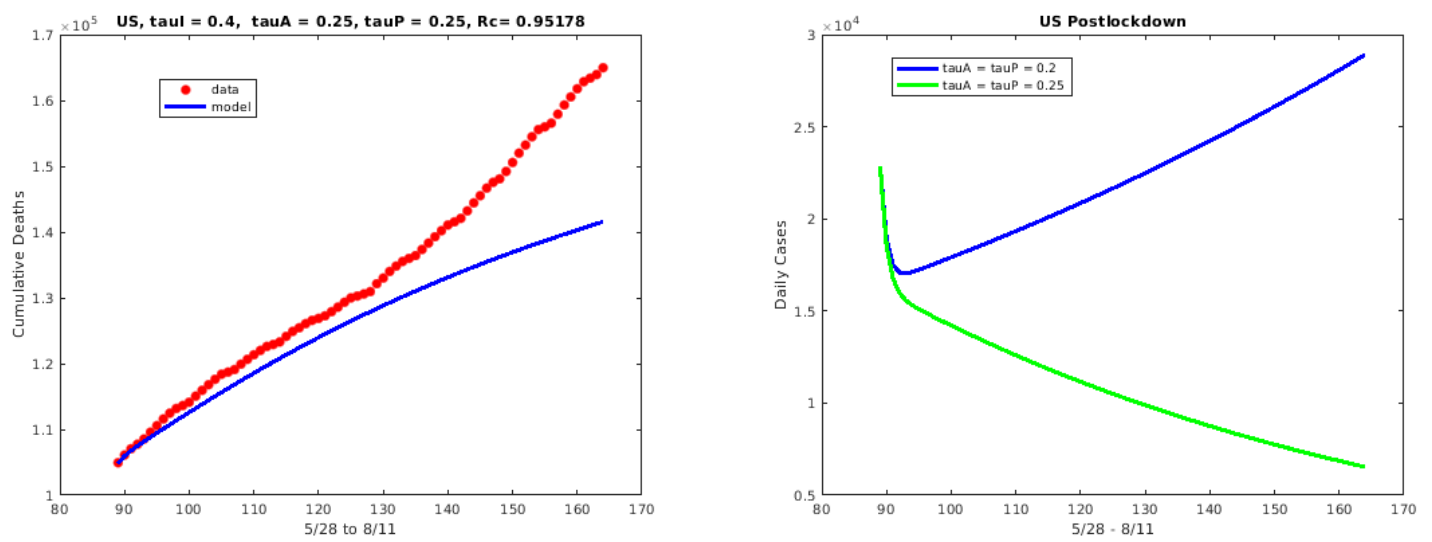

Figure 14: The effect of an increase in $\tau_{A}$ and $\tau_{P}$

\subsection{Forward Simulation of the Pandemic}

Natural herd immunity refers to the process by which enough of the population achieves immunity via natural recovery from the disease, so that the remaining population that is not immune also receives protection against the acquisition of the disease. The process involves a natural herd immunity threshold, that is, the minimum number of people required to achieve disease-acquired immunity. For a disease with a basic reproduction number of $\mathcal{R}_{0}$, the necessary minimum fraction of people who must achieve immunity can be given as $1-1 / \mathcal{R}_{0}$. We simulate for both Wisconsin (a state which was considered an epicenter later in 2020) and the entire US, a scenario in which COVID-19 is allowed to run its natural course (with no implementation of any new control or mitigation strategies) until natural herd immunity is achieved . For both Wisconsin and the entire US, the number of deaths that occur before reaching the herd immunity threshold, is considerably high; thus, making the success of a natural herd immunity strategy for COVID-19 questionable (at the very least). For the state of Wisconsin, the number of deaths that occurs is approximately 80,000(Fig 15A)and for the entire U.S., the number of deaths is more than 5 million (Fig 15B).
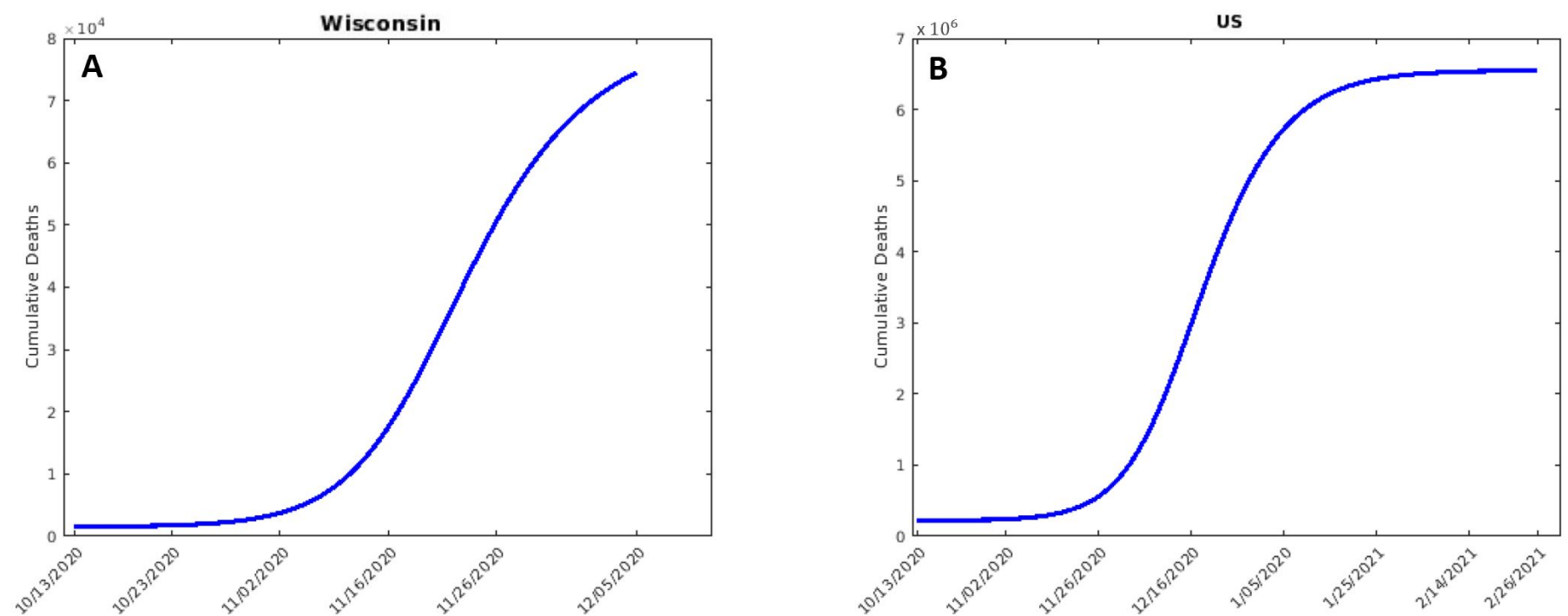

Figure 15: The number of deaths that will occur before natural herd immunity threshold is achieved for the state of Wisconsin (A) and the entire US (B). The simulations are run starting on October 13. 


\section{Summary}

From various perspectives, our analysis indicates that overall, the drivers of COVID-19 are the asymptomatic and pre-symptomatic infectious individuals. Even with vaccines, it is critical to control the spread. We see that if testing is increased, these asymptomatic and pre-symptomatic infectious have a greater impact than the symptomatic infectious; hence, widespread and frequent testing is key to controlling the spread of impact, the benefit of even a short lockdown (2 weeks) in reducing deaths is considerable.

\section{Acknowledgements}

The authors extend special thanks to the 2020 African Diaspora Joint Mathematics Workshop (ADJOINT), Dr. Abba Gumel (our ADJOINT group leader) and the Mathematical Sciences Research Institute (MSRI) - especially the MSRI IT members Aaron Hale \& Nate Orange. We also acknowledge the following funding sources for ADJOINT: Alfred P. Sloan Foundation (G-2020-12602), National Science Foundation (DMS-1915954 and DMS-2016406), and National Security Agency (H98230-20-1-0015).

295 7. Appendix A

7.1. Tables of State Variables and Parameter Values

\begin{tabular}{|c|l|}
\hline State Variable & Description \\
\hline$S$ & $\begin{array}{l}\text { Population of susceptible individuals } \\
\text { Population of non-quarantined exposed individuals (infected but not } \\
\text { showing symptoms and cannot transmit infection; newly-infected but not } \\
\text { infectious) }\end{array}$ \\
\hline$E_{P}$ & Population of pre-symptomatic (infectious) individuals \\
\hline$I$ & Population of symptomatically-infectious individuals \\
\hline$A$ & Population of asymptomatically-infectious individuals \\
\hline$J$ & Population of self-isolated individuals \\
\hline$H$ & Population of hospitalized individuals \\
\hline$R$ & Population of recovered individuals \\
\hline$C$ & Population of individuals in intensive care unit (ICU) \\
\hline$D$ & Population of COVID-19 deceased individuals \\
\hline
\end{tabular}

Table 3: State variables for the Model 1 


\begin{tabular}{|c|c|c|c|c|}
\hline Parameter & Description & Range & Baseline value & References \\
\hline$\beta_{I}$ & $\begin{array}{l}\text { Effective contact rate for symptomatically-infectious } \\
\text { individuals }\end{array}$ & Fitting & Fitting & Fitting \\
\hline$\beta_{A}$ & $\begin{array}{l}\text { Effective contact rate for asymptomatically-infectious } \\
\text { individuals }\end{array}$ & Fitting & Fitting & Fitting \\
\hline$\beta_{P}$ & $\begin{array}{l}\text { Effective contact rate for } \\
\text { pre-symptomatically-infections individuals }\end{array}$ & Fitting & Fitting & Fitting \\
\hline$\epsilon_{m}$ & $\begin{array}{l}\text { Efficacy of face masks to prevent transmission and } \\
\text { acquisition of infection }\left(0<\epsilon_{m} \leq 1\right)\end{array}$ & $0.4-0.6$ & 0.5 & 14] \\
\hline$c_{m}$ & $\begin{array}{l}\text { Compliance in face mask usage in the community } \\
\left(0<c_{m} \leq 1\right)\end{array}$ & $0.0190-0.1835$ & per state & 14] \\
\hline$\sigma_{e}$ & $\begin{array}{l}\text { Progression rate from exposed }(E) \text { to } \\
\text { pre-symptomatic infectious class }\left(E_{P}\right) \text { infectious class }\end{array}$ & $1 / 2.5$ day $^{-1}$ & $1 / 2.5$ day $^{-1}$ & 14] \\
\hline$\sigma_{P}$ & $\begin{array}{l}\text { Progression rate from pre-symptomatic infectious } \\
\text { class }\left(E_{P}\right) \text { to asymptomatically infectious or } \\
\text { symptomatically infectious }\end{array}$ & $1 / 2.5$ day $^{-1}$ & $1 / 2.5$ day $^{-1}$ & [14] \\
\hline$r$ & $\begin{array}{l}\text { Fraction of exposed individuals who show clinical } \\
\text { symptoms at the end of the incubation period }\end{array}$ & $0.4-0.6$ & 0.6 & 13. 10. 6] \\
\hline$\tau_{P}$ & $\begin{array}{l}\text { Rate at which pre-symptomatic infectious individuals } \\
\text { self-isolate }\end{array}$ & Fitting & Fitting & Fitting \\
\hline$\tau_{I}$ & $\begin{array}{l}\text { Rate self-quarantined symptomatically-infectious } \\
\text { humans self-isolate }\end{array}$ & $0.07-0.4681$ day $^{-1}$ & $1 / 2.5$ day $^{-1}$ & 18, 5, 15, 12, 16, 11] \\
\hline$\tau_{A}$ & $\begin{array}{l}\text { Rate at which asymptomatically-infectious humans } \\
\text { self-isolate }\end{array}$ & Fitting & Fitting & Fitting \\
\hline$\gamma_{I}$ & Recovery rate for individuals in the $I$ class & $1 / 30-1 / 3$ day $^{-1}$ & $1 / 7$ day $^{-1}$ & 13. 10. 6] \\
\hline$\gamma_{A}$ & Recovery rate for individuals in the $A$ class & $1 / 14-1 / 3$ day $^{-1}$ & $1 / 7$ day $^{-1}$ & [13. 10. 6 \\
\hline$\gamma_{J}$ & Recovery rate for self-isolated individuals & $0.0714-0.1667$ day $^{-1}$ & $1 / 8$ day $^{-1}$ & [13] \\
\hline$\gamma_{H}$ & Recovery rate for hospitalized patients & $1 / 30-1 / 3$ day $^{-1}$ & $1 / 14$ day $^{-1}$ & 13. 10. 6] \\
\hline$\gamma_{C}$ & Recovery rate for ICU patients & $0.018-0.14$ day $^{-1}$ & 0.0225 day $^{-1}$ & [13. 9] \\
\hline$\psi_{H}$ & Rate of ICU admission for hospitalized individuals & $0.02-0.1667$ day $^{-1}$ & 0.083 day $^{-1}$ & 13. 6. 11 \\
\hline$\alpha_{I}$ & $\begin{array}{l}\text { Hospitalization rate for symptomatically-infectious } \\
\text { individuals }\end{array}$ & $0.1111-0.3333$ day $^{-1}$ & 0.2199 day $^{-1}$ & [6. 8. 10] \\
\hline$\alpha_{A}$ & $\begin{array}{l}\text { Hospitalization rate for asymptomatically-infectious } \\
\text { individuals }\end{array}$ & $0.1667-0.3333$ day $^{-1}$ & $1 / 4$ day $^{-1}$ & 4. 13 \\
\hline$\alpha_{J}$ & Hospitalization rate for self-isolated individuals & Fitting & Fitting & Fitting \\
\hline$\delta_{I}$ & $\begin{array}{l}\text { Disease-induced mortality rate for } \\
\text { symptomatically-infectious individuals }\end{array}$ & $0.001-0.1$ day $^{-1}$ & 0.0225 day $^{-1}$ & 6. 11, 13, 7] \\
\hline$\delta_{A}$ & $\begin{array}{l}\text { Disease-induced mortality rate for } \\
\text { asymptomatically-infectious individuals }\end{array}$ & $0.001-0.1$ day $^{-1}$ & 0.0075 day $^{-1}$ & 6. 7 \\
\hline$\delta_{H}$ & $\begin{array}{l}\text { Disease-induced mortality rate for hospitalized } \\
\text { individuals }\end{array}$ & $0.001-0.1$ day $^{-1}$ & 0.015 day $^{-1}$ & [10. 6. 11] \\
\hline$\delta_{C}$ & Disease-induced mortality rate individuals in ICU & $0.001-0.1$ day $^{-1}$ & 0.0225 day $^{-1}$ & 10. 6. 6] \\
\hline
\end{tabular}

Table 4: Parameter notation, description, values and sources for the Model 1. Some values change depending on the state and time period and for simulations that are post post-lockdown or involve testing implications, the testing parameter values are chosen to moreso align with reported testing trends.
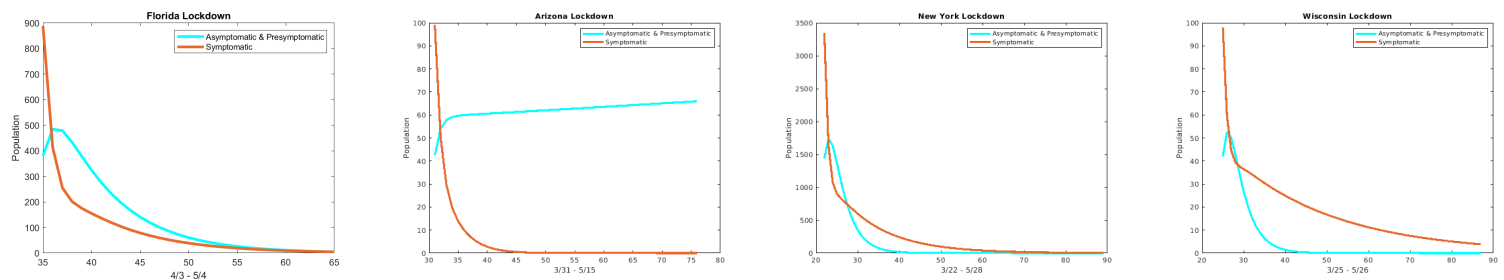

Figure 16: The plots show the daily cases for each state with $\beta_{I}=0$ (cyan curve) and $\beta_{A}=\beta_{P}=0$ (red curve) for the lockdown period.

\subsection{Goodness of Fit}

In this section, we show the plots for the goodness of fit of the lockdown period for each state and the

306 US as a whole. 

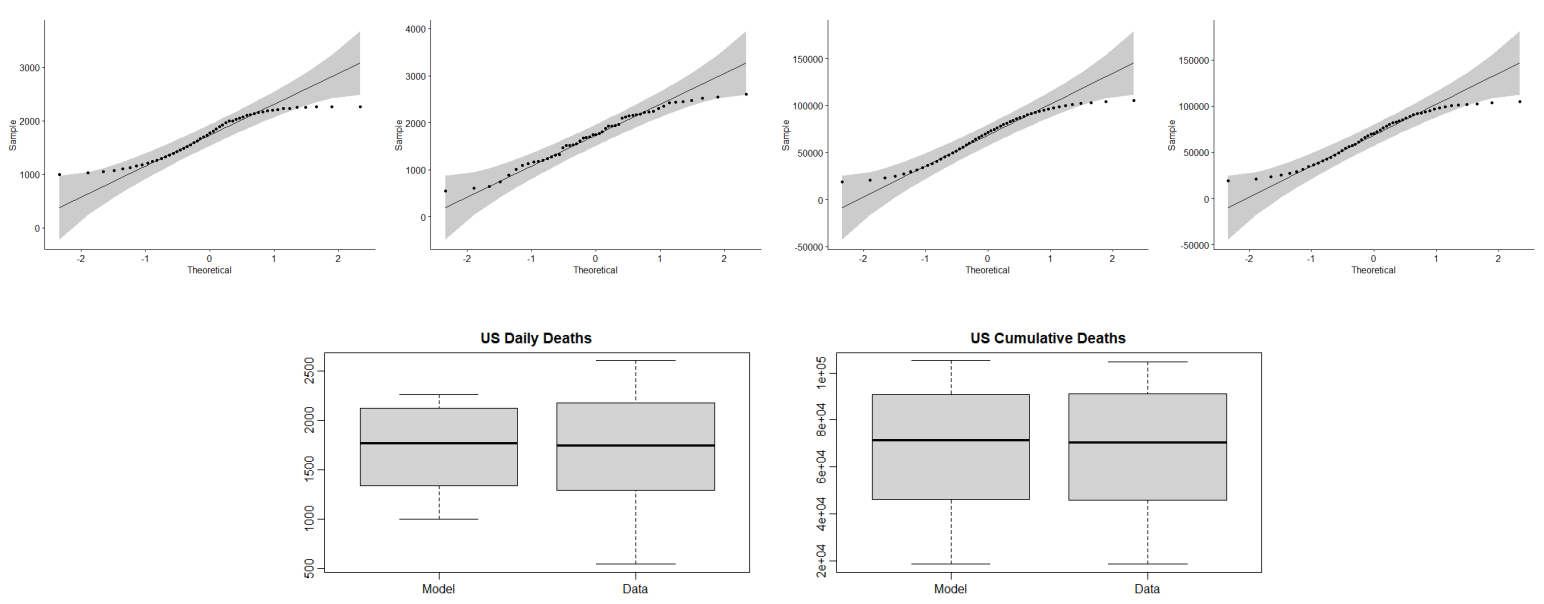

Figure 17: US Row 1: Q-Q plots for the (1) Daily death values from the model (2) Daily death values from the data (3) Cumulative death values from the model (4) Cumulative death values from the data. Row 2: Box plot of the (1) Daily death values from the model and data (2) Cumulative death values from the model and data.
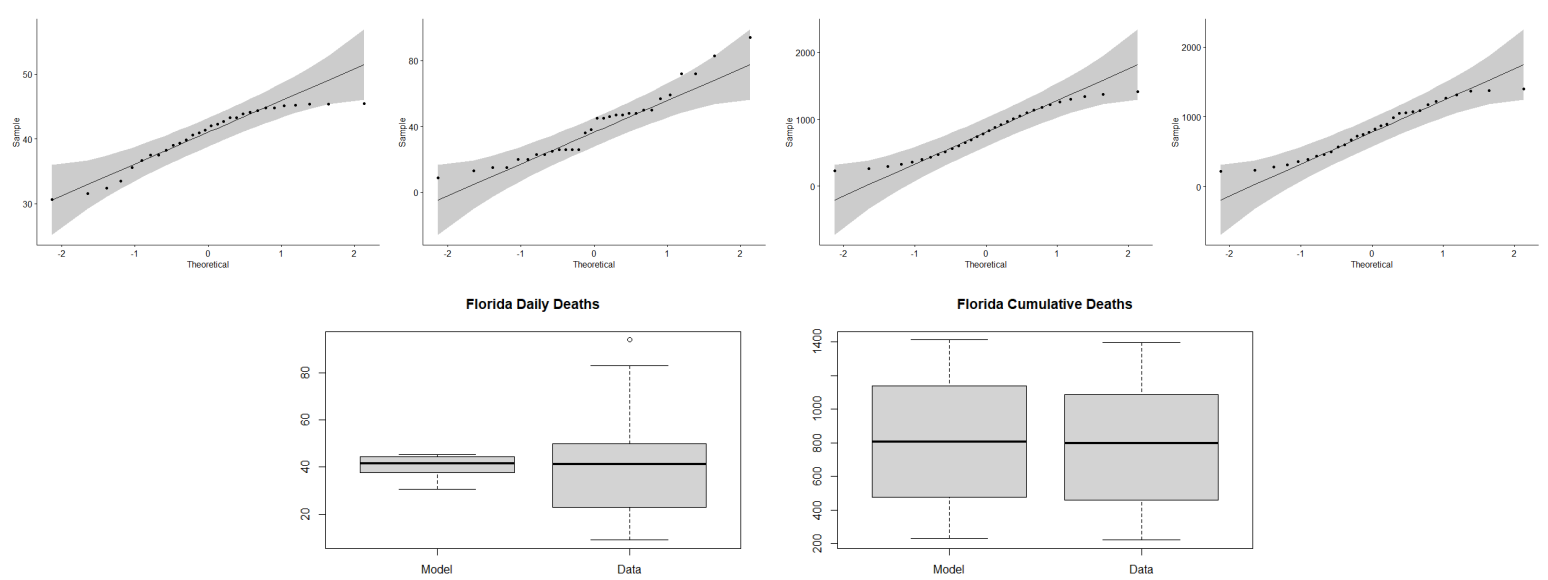

Figure 18: Florida Row 1: Q-Q plots for the (1) Daily death values from the model (2) Daily death values from the data (3) Cumulative death values from the model (4) Cumulative death values from the data. Row 2: Box plot of the (1) Daily death values from the model and data (2) Cumulative death values from the model and data.
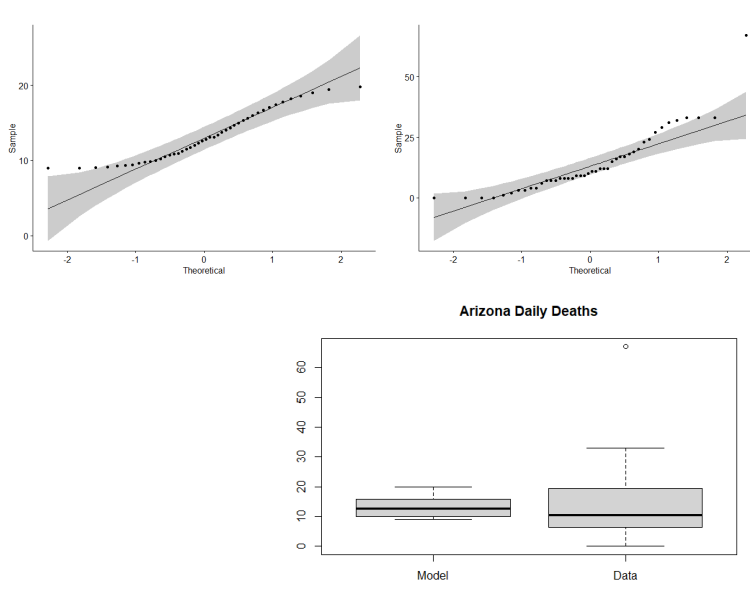
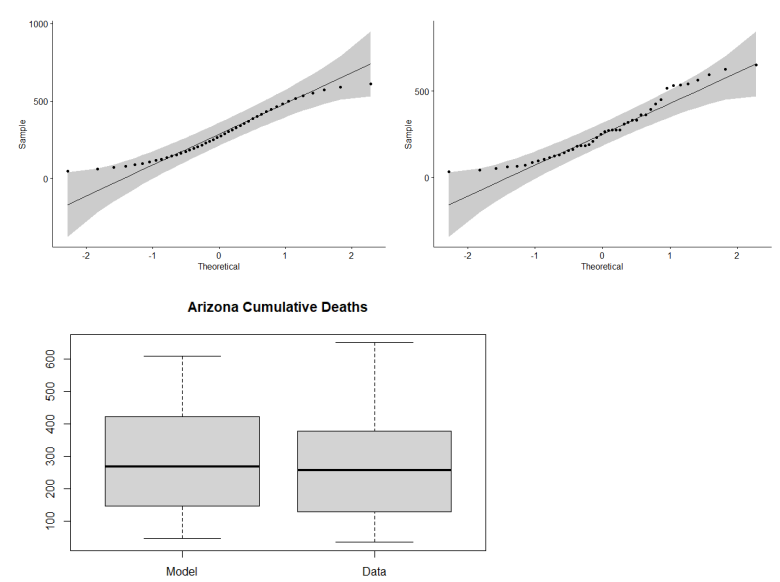

Figure 19: Arizona Row 1: Q-Q plots for the (1) Daily death values from the model (2) Daily death values from the data (3) Cumulative death values from the model (4) Cumulative death values from the data. Row 2: Box plot of the (1) Daily death values from the model and data (2) Cumulative death values from the model and data. 

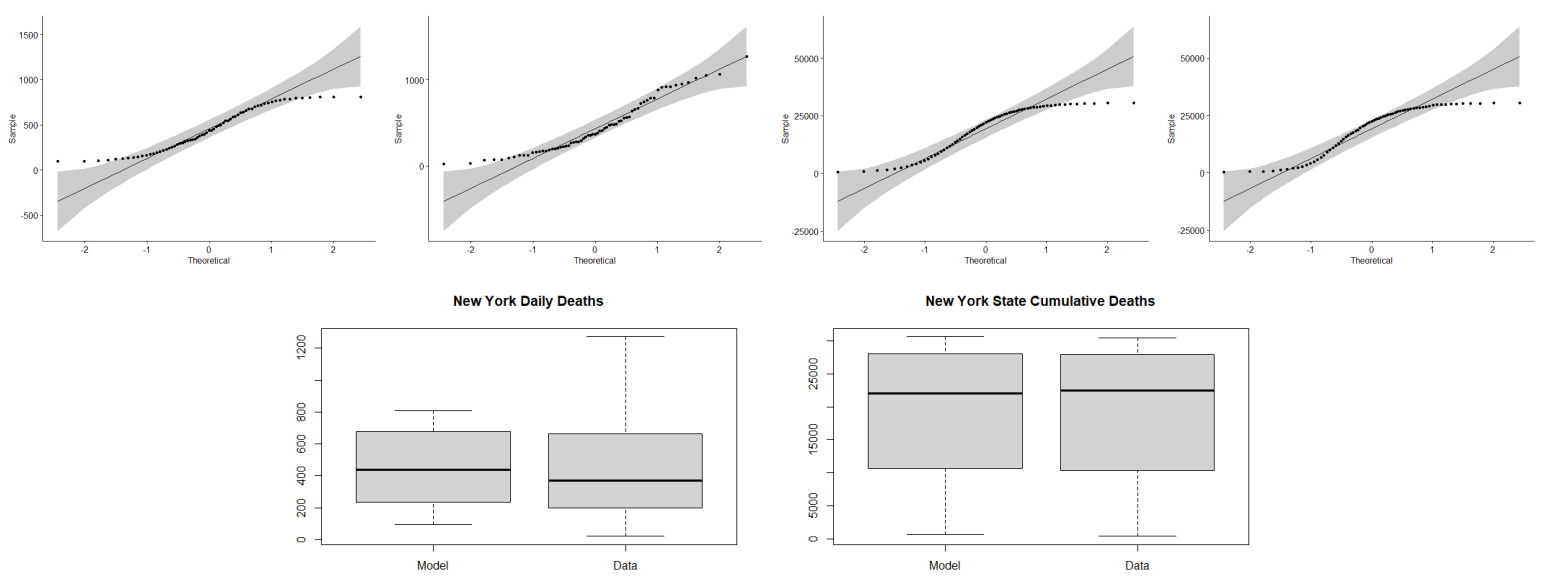

Figure 20: New York Row 1: Q-Q plots for the (1) Daily death values from the model (2) Daily death values from the data (3) Cumulative death values from the model (4) Cumulative death values from the data. Row 2: Box plot of the (1) Daily death values from the model and data (2) Cumulative death values from the model and data.
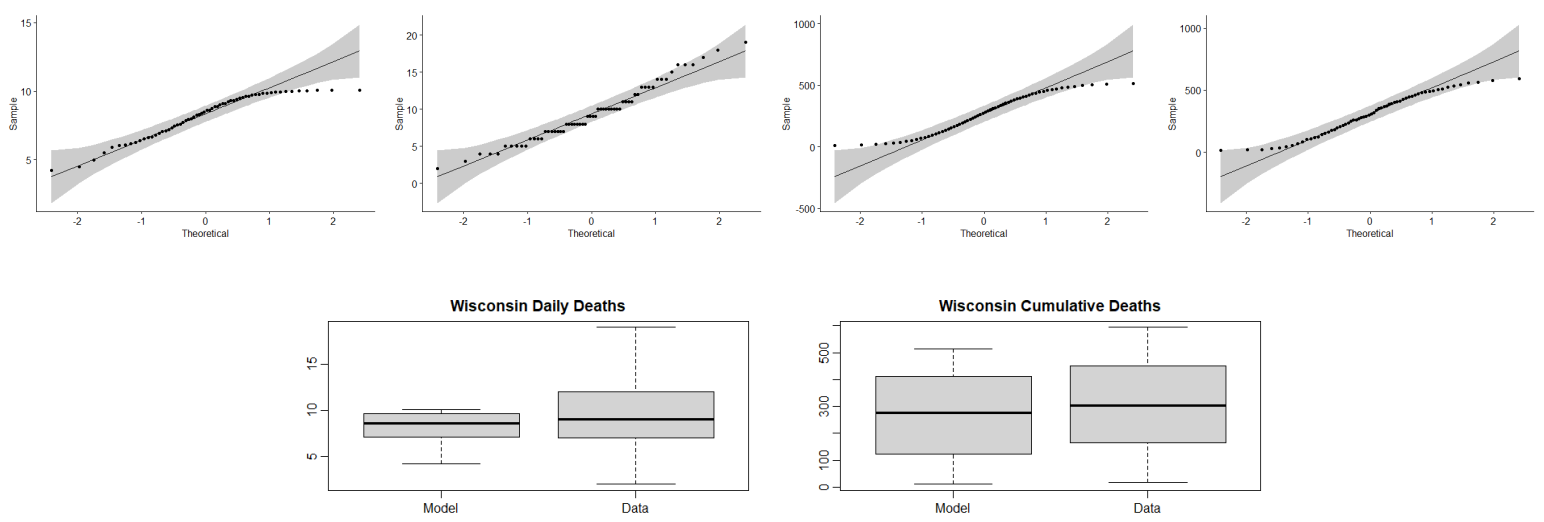

Figure 21: Wisconsin Row 1: Q-Q plots for the (1) Daily death values from the model (2) Daily death values from the data (3) Cumulative death values from the model (4) Cumulative death values from the data. Row 2: Box plot of the (1) Daily death values from the model and data (2) Cumulative death values from the model and data. 
[1] Coronavirus. https://www.cdc.gov/coronavirus/2019-84ncov/hcp/planning-scenarios.html. Accessed: 2020-12-10.

[2] Covid-19 dashboard by the center for systems science and engineering. https://coronavirus.jhu. edu/map.html. Accessed: 2020-12-10.

[3] Covid-19 statistics.

https://covid19.healthdata.org/united-states-of-america?view= mask-use\&tab=trendl. Accessed: 2020-12-10.

[4] Management of patients with confirmed 2019-ncov. https://www.cdc.gov/coronavirus/2019-ncov/ hcp/clinical-guidance-management-patients.html. Accessed: 2020-12-10.

[5] Gerardo Chowell, Diego Chowell, Kimberlyn Roosa, Ranu Dhillon, and Devabhaktuni Srikrishna. Sustainable social distancing through facemask use and testing during the covid-19 pandemic. medRxiv, pages 2020.04.01.20049981-2020.04.01.20049981, 2020.

[6] Steffen E. Eikenberry, Marina Mancuso, Enahoro Iboi, Tin Phan, Keenan Eikenberry, Yang Kuang, Eric Kostelich, and Abba B. Gumel. To mask or not to mask: Modeling the potential for face mask use by the general public to curtail the covid-19 pandemic. Infectious Disease Modelling, 5:293-308, 2020.

[7] N. Ferguson and et. al. Report 9: Impact of non-pharmaceutical interventions (npis) to reduce covid19 mortality and healthcare demand. 2020.

[8] Shikha Garg and et al. Hospitalization Rates and Characteristics of Patients Hospitalized with Laboratory-Confirmed Coronavirus Disease 2019 - COVID-NET, 14 States, March 1 - 30, 2020. Morbidity and Mortality Weekly Report, 2020.

[9] Jeremy Gold and et al. Characteristics and Clinical Outcomes of Adult Patients Hospitalized with COVDI-19 -Georgia, March 2020. Centers for Disease Control and Prevention Morbidity and Mortality Weekly Report, 69.

[10] Abba B. Gumel, Enahoro Iboi, and Calistus N. Ngonghala. Using mathematics to understand covid-19 dynamics. Notices of the American Mathematical Society, (Under review), 2020.

[11] Enahoro Amos Iboi, Calistus N. Ngonghala, and Abba B Gumel. Will an imperfect vaccine curtail the covid-19 pandemic in the u.s.? medRxiv, page 2020.05.10.20097428, 012020.

[12] Adam J Kucharski and et al. Effectiveness of isolation, testing, contact tracing, and physical distancing on reducing transmission of sars-cov-2 in different settings: a mathematical modelling study. The Lancet Infectious Diseases, 062020.

[13] Calistus N. Ngonghala, Enahoro Iboi, Steffen Eikenberry, Matthew Scotch, Chandini Raina MacIntyre, Matthew H. Bonds, and Abba B. Gumel. Mathematical assessment of the impact of non-pharmaceutical interventions on curtailing the 2019 novel coronavirus. Mathematical Biosciences, 325:108364, 2020.

[14] N. Iboi Enahoro A. Gumel Abba B. Ngonghala, Calistus. Could masks curtail the post-lockdown resurgence of COVID-19 in the US? Mathematical Biosciences, 329:1-18, 2020.

[15] Timothy $\mathrm{P}$ Sheahan and et al. An orally bioavailable broad-spectrum antiviral inhibits sars-cov-2 in human airway epithelial cell cultures and multiple coronaviruses in mice. Science Translational Medicine, 12(541):eabb5883-eabb5883, 042020.

[16] Suochen Tian and et al. Clinical Characteristics and Reasons for Differences in Duration From Symptom Onset to Release From Quarantine Among Patients With COVID-19 in Liaocheng, China. 2020. 
[17] P. van den Driessche and J. Watmough. Reproduction numbers and sub-threshold endemic equilibria for compartmental models of disease transmission. Mathematical Biosciences, 180:29-48, 2002.

[18] Lee Worden, Rae Wannier, Seth Blumberg, Alex Y. Ge, George W. Rutherford, and Travis C. Porco. Estimation of effects of contact tracing and mask adoption on covid-19 transmission in san francisco: a modeling study. medRxiv, pages 2020.06.09.20125831-2020.06.09.20125831, 2020. 


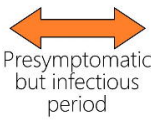


$\delta_{I}$

$\lambda=\left(1-\epsilon_{m} c_{m}\right)\left(\beta_{I} I+\beta_{A} A+\beta_{P} E_{P}\right) \frac{1}{N}$

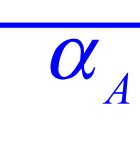




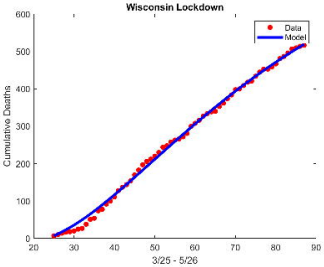




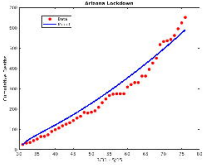


Florida Lockdown

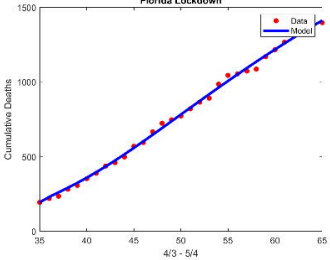




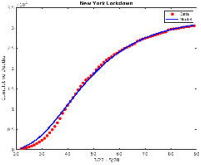




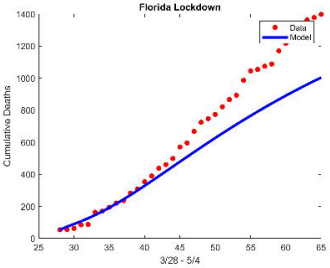




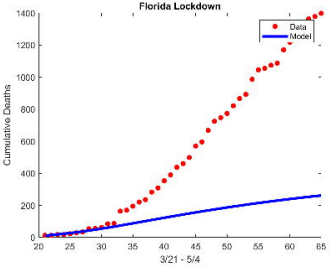




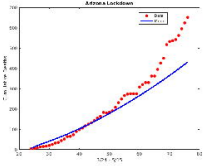




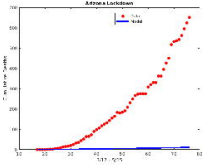




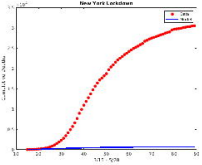




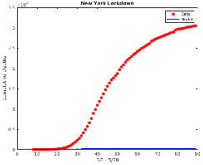




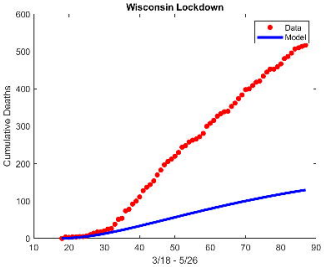




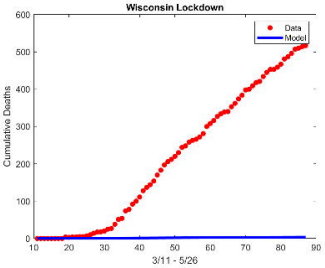




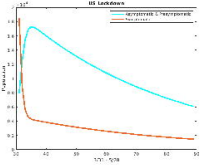


aR Lavelaun

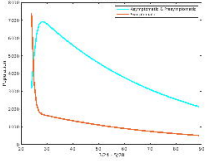




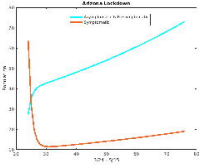




\section{Artransherbaher}

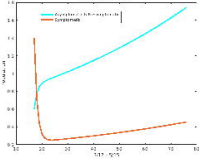




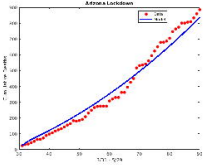




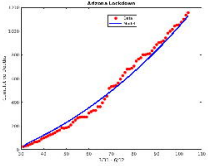




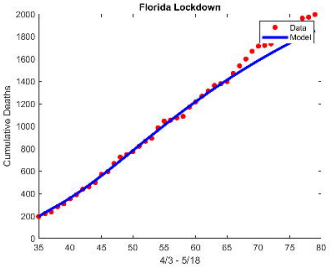




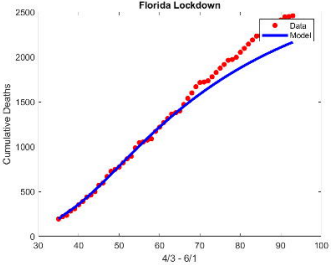




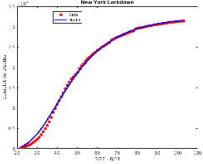




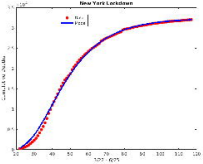


Wheas as Leablewor

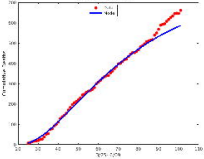


Whawele Lewalaven

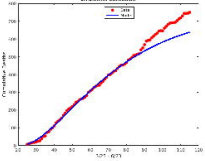




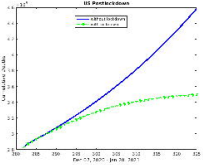




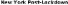

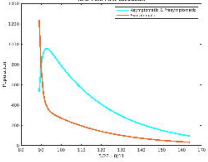


Whanele Lewatavn

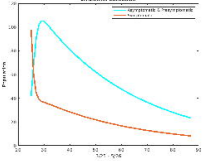




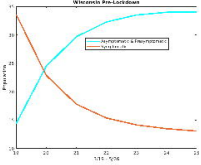


viruasom Pastlectorians

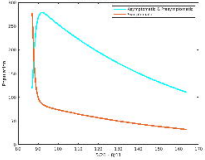


Solrens Prntablaws

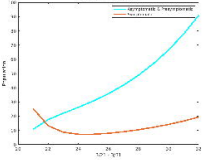




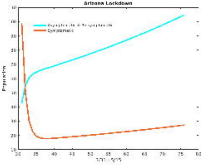




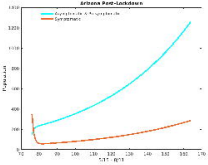


Florida Lockdown

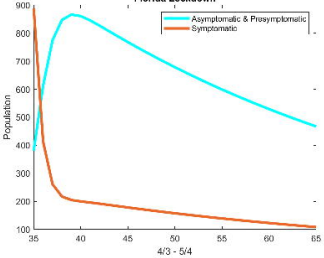




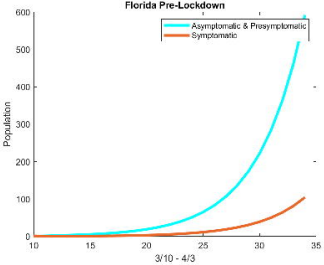




\section{Florida Post-Loekdown}

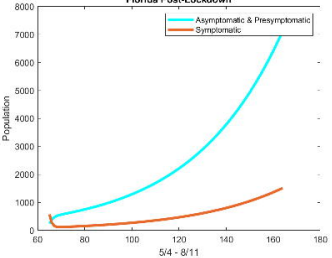




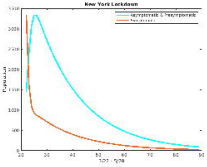


Urw Vuit Pre-Lebalen

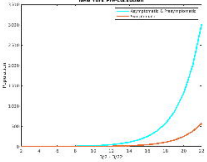




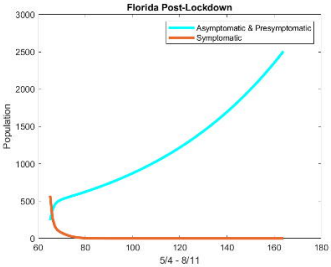


Urw Vuit Pre-Lebalen

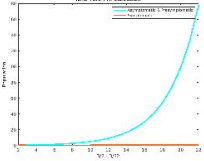


VmwYak Pand-Letabue

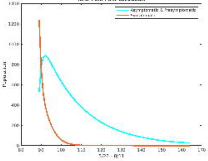




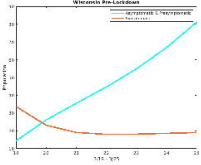


viruasom Pastlectorians

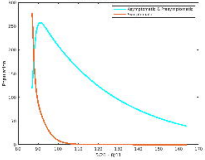


Solrens Prntablaws

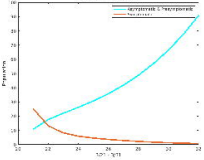




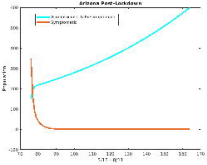




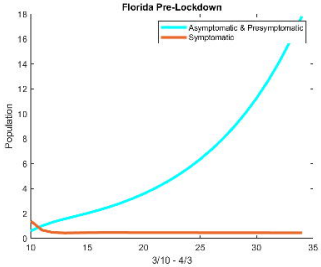




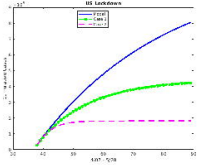




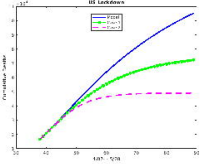




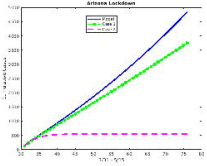




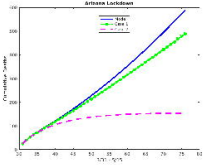




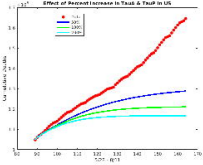




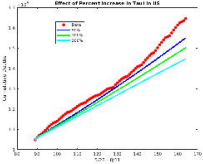




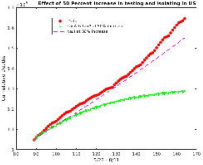




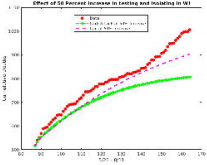




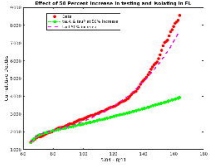




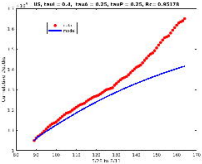




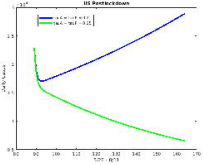


Florida Lockdown

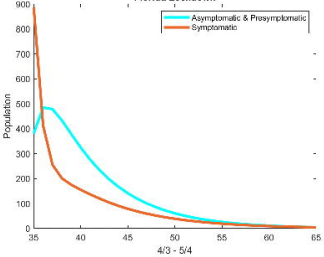




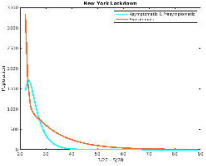


Whanele Lewatavn

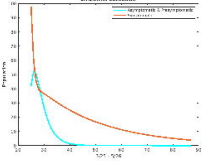




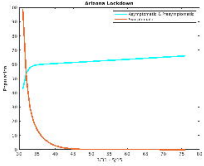




\section{us Cuntulative Deaths}

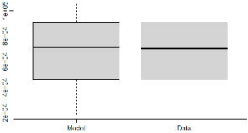




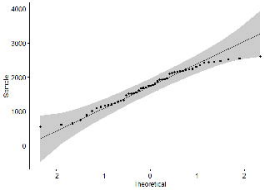




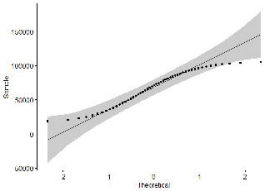




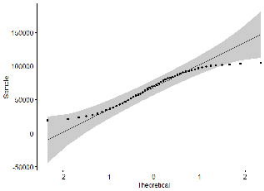




\section{US Daly Deathe}

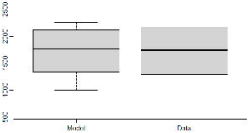


Finrida Cumulative Deaths
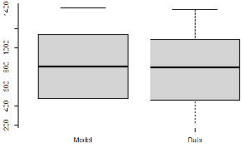
Florida Daly Dasthe

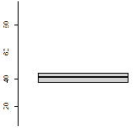

ret:da1

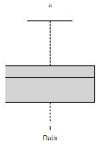


$\mathbf{y}$
$\overline{\mathbf{w}}$
$\mathrm{i}$

$$
\text { 56- }
$$

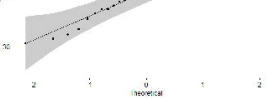




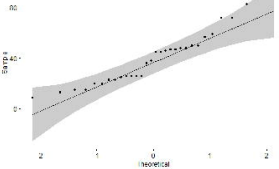


zove -

$\frac{e^{n}}{n}$

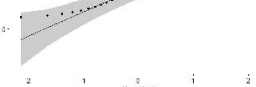

Inacribes 
2000 -

1 $120 \mathrm{c}-$

$\frac{a}{0}$

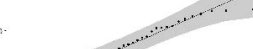

t.

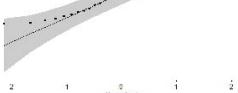

Inacribec 


\section{Arizona Cumulative Dasthe}
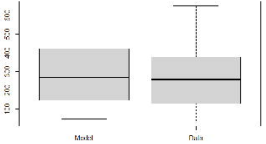
20

14

$\frac{1}{2}$

is

10

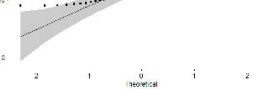


모.

$\overline{0}=$

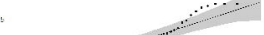

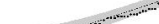

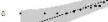

*3

1

U

2

hoorecsl 
0
$\overline{0}$
13

500 -

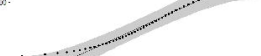




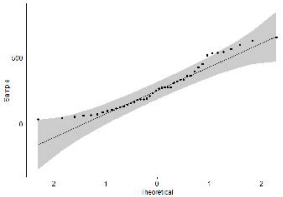




\section{Arizona Daily Deathe}

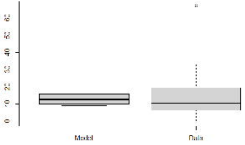




\section{Now York State Cumulativa Deaths}

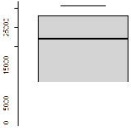

ret:ds

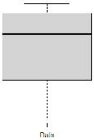


150. -

1000 -

1

$\frac{a}{p}$ bou-

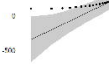

2

v

2

Irocribec 
$\frac{1}{9}$

$1 \mathrm{nn}$ : -

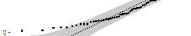

2

v

?

Inocribec 


\section{entat -}

a. 24.70 :

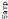

-7.50\%:

$2 \quad 1 \quad \frac{\dot{0}}{1100 \mathrm{stico}}$


sove:-

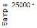

-7.500 ?

$2 \quad 1 \quad \frac{\dot{0}}{1100 \mathrm{stico}}$


Now York Daily Deathe

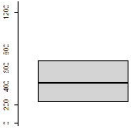

restas

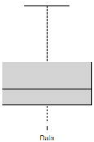




\section{wisccnsin pary peains}

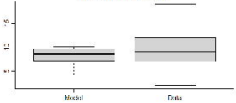


wsconsIn cumuledve deeths

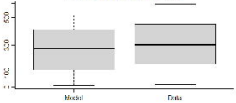


11. -

$\frac{5}{5}^{10}$

$\begin{array}{llll}2 & 1 & i & i\end{array}$




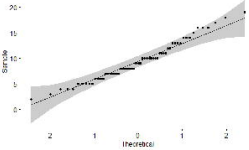


$100:$

50

$\frac{2}{5}$

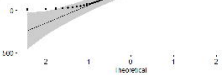


1fan

500

$\frac{x}{5}$

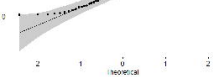


